Article

\title{
The Sum of a Finite Group of Weights of a Hopf Algebra
}

\section{Apoorva Khare}

Department of Mathematics and Department of Statistics, Stanford University, 390 Serra Mall, Stanford, CA 94305, USA; E-Mail: khare @ stanford.edu; Tel.: +1-650-723-2957;

Fax: +1-650-725-8977

Received: 2 July 2012; in revised form: 17 September 2012 / Accepted: 17 September 2012 /

Published: 5 October 2012

\begin{abstract}
Motivated by the orthogonality relations for irreducible characters of a finite group, we evaluate the sum of a finite group of linear characters of a Hopf algebra, at all grouplike and skew-primitive elements. We then discuss results for products of skew-primitive elements. Examples include groups, (quantum groups over) Lie algebras, the small quantum groups of Lusztig, and their variations (by Andruskiewitsch and Schneider).
\end{abstract}

Keywords: Hopf algebra; weights; grouplike; skew-primitive

Classification: MSC 16W30 (primary), 17B10 (secondary)

\section{Introduction}

\subsection{Motivation}

Suppose $G$ is a finite group, with irreducible characters $\widehat{G}=\{\chi: \mathbb{C} G \rightarrow \mathbb{C}\}$, over $\mathbb{C}$. As is well known, they satisfy the orthogonality relations. Here is a consequence: if one defines $\theta_{\chi}:=\frac{1}{|G|} \sum_{g \in G} \chi\left(g^{-1}\right) g \in H=\mathbb{C} G$, then $\nu\left(\theta_{\chi}\right)=0$ for $\chi \neq \nu \in \widehat{G}$. Similarly, the other orthogonality relation (for columns) implies that $\sum_{\chi \in \widehat{G}}\left(\operatorname{dim} \rho_{\chi}\right) \chi(g)$ is either zero or a factor of $|G|$, depending on whether or not $g=1$. (Here, $\rho_{\chi}$ is the irreducible representation with character $\chi$.)

In what follows, we work over a commutative integral domain $R$. Note that there is an analogue of the first orthogonality relation for any $R$-algebra $H$ that is a free $R$-module. Namely, given a linear character (i.e., algebra map or weight) $\lambda: H \rightarrow R$, define a left $\lambda$-integral of $H$ to be any (nonzero) $\Lambda_{L}^{\lambda} \in H$ so that $h \Lambda_{L}^{\lambda}=\lambda(h) \Lambda_{L}^{\lambda}$ for all $h \in H$. One can similarly define right and two-sided $\lambda$-integrals in $H$. (For 
instance, the $\theta_{\chi}$ 's above are two-sided $\chi$-integrals for any weight $\chi$.) Then the following result holds in any algebra:

Lemma 1.1. If $\lambda \neq \nu$ are weights of $H$ with corresponding nonzero left integrals $\Lambda_{L}^{\lambda}, \Lambda_{L}^{\nu}$ respectively, then $\nu\left(\Lambda_{L}^{\lambda}\right)=0=\Lambda_{L}^{\lambda} \Lambda_{L}^{\nu}$.

Proof. Choose $h$ so that $\lambda(h) \neq \nu(h)$. Then

$$
\nu(h) \nu\left(\Lambda_{L}^{\lambda}\right) \Lambda_{L}^{\nu}=\nu(h) \Lambda_{L}^{\lambda} \Lambda_{L}^{\nu}=h \Lambda_{L}^{\lambda} \Lambda_{L}^{\nu}=\left(h \Lambda_{L}^{\lambda}\right) \Lambda_{L}^{\nu}=\lambda(h) \Lambda_{L}^{\lambda} \Lambda_{L}^{\nu}=\lambda(h) \nu\left(\Lambda_{L}^{\lambda}\right) \Lambda_{L}^{\nu}
$$

so $\nu\left(\Lambda_{L}^{\lambda}\right)(\lambda(h)-\nu(h)) \Lambda_{L}^{\nu}=0$. Since we are working over an integral domain and within a free module, this implies that $\nu\left(\Lambda_{L}^{\lambda}\right)=0$. Moreover, $\Lambda_{L}^{\lambda} \Lambda_{L}^{\nu}=\nu\left(\Lambda_{L}^{\lambda}\right) \Lambda_{L}^{\nu}=0$.

It is now natural to seek a "Hopf-theoretic" analogue for the second orthogonality relation (which might involve only weights, and not all irreducible characters). Note that in general, a Hopf algebra might not have a nontrivial (sub)group of linear characters that is finite: for instance, $U(\mathfrak{g})$ for a complex Lie algebra $\mathfrak{g}$. However, if such subgroups do exist, then we attempt to evaluate the sum of all weights in the subgroup (i.e., the coefficient of each weight is $\operatorname{dim} \rho=1$ ), at various elements of $H$. For example, one may ask: does this sum always vanish at a nontrivial grouplike $g \in H$ ?

This problem is also interesting from another perspective. Given a Hopf algebra $H$, it is interesting to seek connections between the representations of $H$ and those of $H^{*}$ (or, the comodules over $H^{*}$ and over $H$ respectively). A famous example where both of these come into play is the well-known formula due to Radford [1] concerning the antipode. The setting of this paper is another such case: note that the weights of $H$ are precisely the one-dimensional representations of $H^{*}$ (or the group-like elements of $H^{*}$ ), while grouplike and skew-primitive elements in a Hopf algebra $H$ correspond to the 1-dimensional and non-semisimple 2-dimensional $H$-comodules (or $H^{*}$-modules) respectively. The present paper explores the interplay between these objects.

\subsection{One of the Setups, and Some References}

Instead of attempting a summary of the results, which are several and computational, we make some remarks. Given an $R$-algebra $A$, let $\Gamma_{A}$ denote the set of weights of $A$. (If $H$ is a Hopf algebra, then $\Gamma_{H}$ is a group.) Let $\Pi \subset \Gamma_{H}$ be a finite group of weights of a Hopf algebra $H$ over a commutative unital integral domain $R$. One has the notion of grouplike elements (i.e., $\Delta(g)=g \otimes g$ ) and skew-primitive elements in $H$.

The first step is to compute $\Sigma_{\Pi}$ at all grouplike and skew-primitive elements in $H$, where $\Sigma_{\Pi}:=\sum_{\gamma \in \Pi} \gamma: H \rightarrow R$. In a wide variety of examples-including finite-dimensional pointed Hopf algebras [2] - the computations reduce to grouplike elements. In other words, there are several families of algebras, where knowing $\Sigma_{\Pi}$ at grouplike elements effectively tells us $\Sigma_{\Pi}$ at all elements.

The next objective is to evaluate $\Sigma_{\Pi}$ at products of skew-primitive elements. Once again, in the spirit of the previous paragraph, there are numerous examples of Hopf algebras generated by grouplike and skew-primitive elements in the literature. The first two examples below are from folklore, and references can be found in [3]. 
1. By the Cartier-Kostant-Milnor-Moore Theorem (e.g., see [3, Theorem 5.6.5]), every cocommutative connected Hopf algebra $H$ over a field of characteristic zero, is of the form $U(\mathfrak{g})$, where $\mathfrak{g}$ is the set of primitive elements in $H$. Similarly, every complex cocommutative Hopf algebra is generated by primitive and grouplike elements.

2. If the Hopf algebra is pointed (and over a field), then by the Taft-Wilson Theorem [3, Theorem 5.4.1.1], our results can evaluate $\Sigma_{\Pi}$ on any element of $C_{1}$, the first term in the coradical filtration (which is spanned by grouplike and skew-primitive elements).

3. The final example is from a recent paper [2]. The Classification Theorem 0.1 says, in particular, that if $H$ is a finite-dimensional pointed Hopf algebra over an algebraically closed field of characteristic zero, and the grouplike elements form an Abelian group of order coprime to 210, then $H$ is generated by grouplike and skew-primitive elements, and is a variation of a small quantum group of Lusztig.

We conclude this section with one of our results. Say that an element $h \in H$ is pseudo-primitive with respect to $\Pi$ if $\Delta(h)=g \otimes h+h \otimes g^{\prime}$ for grouplike $g, g^{\prime}$ satisfying $\gamma(g)=\gamma\left(g^{\prime}\right)$ for all $\gamma \in \Pi$.

Theorem 1.2. Fix $n \in \mathbb{N}$, as well as the R-Hopf algebra $H$ and a finite subgroup of weights $\Pi$ of $H$. Suppose $h_{1}, \ldots, h_{n} \in H$ are pseudo-primitive with respect to $\Pi$, and $\Delta\left(h_{i}\right)=g_{i} \otimes h_{i}+h_{i} \otimes g_{i}^{\prime}$ for all $i$. Define $\mathbf{h}=\prod_{i} h_{i}$, and similarly, $\mathbf{g}, \mathbf{g}^{\prime}$.

1. If $\operatorname{char}(R)=0$ or $\operatorname{char}(R) \nmid|\Pi|$, then $\Sigma_{\Pi}(\mathbf{h})=0$.

2. Suppose $0<p=\operatorname{char}(R)$ divides $|\Pi|$, and $\Pi_{p}$ is any $p$-Sylow subgroup. If $\Pi_{p} ¥(\mathbb{Z} / p \mathbb{Z})^{m}$ for any $m>0$, then $\Sigma_{\Pi}(\mathbf{h})=0$.

3. (p as above.) Define $\Phi:=\Pi /[\Pi, \Pi]$, and by above, suppose $\Phi_{p} \cong(\mathbb{Z} / p \mathbb{Z})^{k}$ is a $p$-Sylow subgroup of $\Phi$. Let $\Phi^{\prime}$ be any Hall complement(ary subgroup); thus $\left|\Phi^{\prime}\right|=|\Phi| /\left|\Phi_{p}\right|$. Then

$$
\Sigma_{\Pi}(\mathbf{h})=|[\Pi, \Pi]| \cdot \Sigma_{\Phi^{\prime}}(\mathbf{g}) \cdot \Sigma_{\Phi_{p}}(\mathbf{h})
$$

4. If $\Sigma_{\Phi_{p}}(\mathbf{h})$ is nonzero, then $(p-1) \mid n$, and $0 \leq k \leq n /(p-1)$. (Moreover, examples exist wherein $\Sigma_{\Phi_{p}}(\mathbf{h})$ can take any value $r \in R$.)

These results occur below as Proposition 5.5, and Theorems 7.8, 7.5, and 7.10 respectively.

\subsection{Organization}

We quickly explain the organization of this paper. In Section 2, we compute $\Sigma_{\Pi}$ at all grouplike elements in a Hopf algebra. This turns out to be extremely useful in computing $\Sigma_{\Pi}$ in a large class of examples. More precisely, many algebras $A$ that are well-studied in the literature contain a Hopf (sub)algebra $H$ and whose sets of weights are subsets of $\Gamma_{H}$ and kill a large subspace of $A$. These examples include quantum groups, the quantum Virasoro algebra, and finite-dimensional pointed Hopf algebras.

In Section 3, we evaluate $\Sigma_{\Pi}$ at all skew-primitive elements of $H$. This is followed by a brief remark concerning the "degenerate" example of quiver (co)algebras.

Computing $\Sigma_{\Pi}$ at all products of skew-primitive elements is a difficult problem. We show in the next Section 4 that on occasion, it can be reduced to computing $\Sigma_{\Gamma_{H} /\left[\Gamma_{H}, \Gamma_{H}\right]}$. In Section 6, we are able to 
obtain results when $\Gamma_{H}$ itself is Abelian. These computations are useful in working with products of special kinds of skew-primitive elements in other sections.

In the rest of the paper, we work with "pseudo-primitive elements" $h_{i}$ in order to obtain more detailed results. In Section 5, we show that $\Sigma_{\Pi}\left(h_{1} \ldots h_{n}\right)=0$ whenever $\operatorname{char}(R) \nmid|\Pi|$. In Section 7, we study the case when $\operatorname{char}(R)=p$ divides $|\Pi|$. In this case, there are severe restrictions on the $p$-Sylow subgroup of $\Pi$, in order for $\Sigma_{\Pi}(\mathbf{h})$ to be nonzero; moreover, we write down a result that helps compute $\Sigma_{\Pi}(\mathbf{h})$.

We conclude with a detailed study of further examples_Lie algebras, degenerate affine Hecke algebras of reductive type, and then a Hopf algebra generated by grouplike and skew-primitive elements, where $\Sigma_{\Pi}(\mathbf{h})$ can take on all values.

\section{Grouplike Elements and Quantum Groups}

\subsection{Preliminaries}

We first set some notation, and make some definitions.

Definition 2.1. Suppose $R$ is a commutative unital integral domain.

1. Integers in $R$ are the image of the group homomorphism $\mathbb{Z} \rightarrow R$, sending $1 \mapsto 1$.

2. A weight of an $R$-algebra $H$ is an $R$-algebra map : $H \rightarrow R$. Denote the set of weights by $\Gamma_{H}$. Occasionally we will also use $\Gamma=\Gamma_{H}$. Given $\nu \in \Gamma_{H}$, the $\nu$-weight space of an $H$-module $V$ is $V_{\nu}:=\{v \in V: h \cdot v=\nu(h) v \forall h \in H\}$.

3. Given a left $R$-module $H$, define $H^{*}:=\operatorname{Hom}_{R-\bmod }(H, R)$.

4. An $R$-Hopf algebra $H$ is an $R$-algebra $(H, \mu=\cdot, \eta)$ (where $\mu, \eta$ are coalgebra maps) that is also an $R$-coalgebra $(H, \Delta, \varepsilon)$ (where $\Delta, \varepsilon$ are algebra maps), further equipped with an antipode $S$ (which is an $R$-(co)algebra anti-homomorphism).

5. In a Hopf algebra (or a bialgebra), an element $h$ is grouplike if $\Delta(h)=h \otimes h$, and primitive if $\Delta(h)=1 \otimes h+h \otimes 1$. Define $G(H)$ (respectively $H_{\text {prim }}$ ) to be the set of grouplike (respectively primitive) elements in a Hopf algebra $H$.

There are several standard texts on Hopf algebras; for instance, see [3-6]. In particular, since $H$ is a coalgebra, $H^{*}$ is also an $R$-algebra under convolution $\Delta^{*}$ : given $\lambda, \nu \in H^{*}$ and $h \in H$, one defines $\langle\lambda * \nu, h\rangle:=\langle\lambda \otimes \nu, \Delta(h)\rangle$. By [4, Theorem 2.1.5] (also see [6, Lemma 4.0.3]), the set $\Gamma_{H}$ of weights is now a group under $*$, with inverse given by $\left\langle\lambda^{-1}, h\right\rangle:=\langle\lambda, S(h)\rangle$.

Note that for an algebra $H$ over a field $k$, the dual space $H^{*}$ is not a coalgebra in general. However, define $H^{\circ}$ to be the set of linear functionals $f: H \rightarrow k$ whose kernel contains an ideal of finite $k$ codimension. Then by [5, Proposition 1.5.3 and Remark 1.5.9], $H^{\circ}$ is a coalgebra whose set of grouplike elements is precisely $\Gamma_{H}$.

Standing Assumption 2.2. For this article, $H$ is any Hopf algebra over a commutative unital integral domain $R$. Fix a finite subgroup of weights $\Pi \subset \Gamma=\Gamma_{H}$.

In general, given a finite subgroup $\Pi \subset \Gamma_{H}$ for any Hopf algebra $H$, the element $\Sigma_{\Pi}:=\sum_{\gamma \in \Pi} \gamma$ is a functional in $H^{*}$, and if $R$ (i.e., its quotient field) has characteristic zero, then $\Sigma_{\Pi}$ does not kill the 
scalars $\eta(R)$. What, then, is its kernel? How about if $\Pi$ is cyclic, or all of $\Gamma_{H}$ (this, only if $H$ is $R$-free, and finite-dimensional over the quotient field of $R$ )?

Lemma 2.3. Suppose $H$ is a Hopf algebra, and $\Pi \subset \Gamma_{H}$ is a finite subgroup of weights.

1. $\Sigma_{\Pi}(1)=0$ if and only if $\operatorname{char}(R)$ divides $|\Pi|$.

2. $[H, H] \subset \operatorname{ker} \Sigma_{\Pi}$.

3. $\Sigma_{\Pi}\left(\operatorname{ad} h\left(h^{\prime}\right)\right)=\varepsilon(h) \Sigma_{\Pi}\left(h^{\prime}\right)$ for all $h, h^{\prime} \in H$. In particular, if $h \in \operatorname{ker} \varepsilon$, then $\operatorname{im}(\operatorname{ad} h) \subset \operatorname{ker} \Sigma_{\Pi}$.

Here, ad stands for the usual adjoint action of $H$. In other words, ad $h\left(h^{\prime}\right):=\sum h_{(1)} h^{\prime} S\left(h_{(2)}\right)$, where we use Sweedler notation: $\Delta(h)=\sum h_{(1)} \otimes h_{(2)}$.

Proof. The first part is easy, and the other two follow because the statements hold if $\Sigma_{\Pi}$ is replaced by any (algebra map) $\gamma \in \Gamma_{H}$.

The goal of this section and the next is to evaluate $\Sigma_{\Pi}$ at all grouplike and skew-primitive elements in $H$. For these computations, a key fact to note is that for all $\lambda \in \Pi$, the following holds in the $R$-algebra $H^{*}$ :

$$
\lambda * \Sigma_{\Pi}=\sum_{\nu \in \Pi} \lambda * \nu=\Sigma_{\Pi}=\cdots=\Sigma_{\Pi} * \lambda
$$

Remark 2.5. We occasionally compute $\Sigma_{\Pi}$ with $H$ an $R$-algebra (that is not a Hopf algebra), where $\Pi \subset \Gamma_{H}$ has a group structure on it. As seen in Proposition 2.13 below, there is an underlying Hopf algebra in some cases.

Definition 2.6. Suppose we have a subset $\Theta \subset \Gamma=\Gamma_{H}$, and $\lambda \in \Pi$.

1. For $g \in G(H)$, set $\Gamma_{g}:=\{\gamma \in \Gamma: \gamma(g)=1\}$, and $\Theta_{g}:=\Gamma_{g} \cap \Theta$.

2. $G_{\Theta}(H)$ is the set (actually, normal subgroup) of grouplike elements $g \in G(H)$ so that $\gamma(g)=1$ for all $\gamma \in \Theta$.

3. For finite $\Theta$, the functional $\Sigma_{\Theta} \in H^{*}$ is given by $\Sigma_{\Theta}:=\sum_{\gamma \in \Theta} \gamma$. Also set $\Sigma_{\emptyset}:=0$.

4. $n_{\lambda}:=o_{\Pi}(\lambda)=|\langle\lambda\rangle|$ is the order of $\lambda$ in $\Pi$.

\section{Remark 2.7.}

1. For instance, $G_{\{\varepsilon\}}(H)=G(H)$, and $G(H) \cap\left[1+\operatorname{im}\left(\mathrm{id}-S^{2}\right)\right] \subset G_{\Gamma}(H)$ because every $\gamma \in \Gamma=$ $\Gamma_{H}$ equals $\left(\gamma^{-1}\right)^{-1}$. This follows since from earlier in this section, $\Gamma_{H}$ is a group with unit $\varepsilon$ and inverse given by $\gamma \mapsto \gamma \circ S$.

2. For any $g \in G(H)$ and $\Theta \subset \Gamma_{H}, \Theta_{g}=\Theta$ if and only if $g \in G_{\Theta}(H)$.

3. $\Theta_{g}$ is a subgroup if $\Theta$ is.

\subsection{Grouplike Elements}

We first determine how $\Sigma_{\Pi}$ acts on grouplike elements, and answer the motivating question above of finding a Hopf-theoretic analogue of the second orthogonality relations for group characters.

Proposition 2.8 ("Orthogonality" at grouplike elements). If $g \in G(H) \backslash G_{\Pi}(H)$, then $\Sigma_{\Pi}(g)=0$. If $g \in G_{\Pi}(H)$, then $\Sigma_{\Pi}(g)=|\Pi|$. 
Proof. To show the first part, apply Equation (2.4) to $g$, with $\lambda \notin \Gamma_{g}$. The second part is obvious.

We now introduce some notation, which is used in discussing several examples.

Definition 2.9. Let $R$ be a commutative unital integral domain, and $l$ be a nonnegative integer.

1. $\sqrt{1}$ (respectively $\sqrt[l]{1}$ ) is the set of $\left(l^{\text {th }}\right)$ roots of unity in $R$. (Thus, $\sqrt[0]{1}=R^{\times}, \sqrt[1]{1}=\{1\}$, and $\sqrt{1}=\cup_{l>0} \sqrt[l]{1}$.)

2. Given $q \in R^{\times}$, $\operatorname{char}(q)$ is the smallest positive integer $m$ so that $q^{m}=1$, and zero if no such $m$ exists.

3. The group $\mathfrak{G}_{n, l}$ is the Abelian group generated by $\left\{K_{i}: 1 \leq i \leq n\right\}$, with relations $K_{i} K_{j}=K_{j} K_{i}, K_{i}^{l}=1$.

4. The group $\mathfrak{G}_{n, l}^{*}$ is defined to be $(\sqrt{1} \cap \sqrt[l]{1})^{n}$.

Thus, $\mathfrak{G}_{n, l}$ is free if and only if $l=0, \mathfrak{G}_{n, l}^{*}=(\sqrt[l]{1})^{n} \forall l>0$, and $\mathfrak{G}_{n, 0}^{*}=(\sqrt{1})^{n}$.

In light of the motivation, the first example where we apply the above result is:

Example 2.10 (Group rings). The above result computes $\Sigma_{\Pi}$ on all of $H$, if $H$ is a group ring. We present a specific example: $G=\mathfrak{G}_{n, l}$ (defined above), for (a fixed) $n \in \mathbb{N}$ and $l \geq 0$. Then $\Gamma=(\sqrt[l]{1})^{n}$, and any finite order element $\gamma \in \Gamma$ maps each $K_{i}$ to a root of unity in $R$. Thus, $\Pi \subset(\sqrt{1})^{n} \cap \Gamma=\mathfrak{G}_{n, l}^{*}$.

We now compute $\Sigma_{\Pi}(g)$ for some $g=\prod_{i=1}^{n} K_{i}^{n_{i}}$, where $n_{i} \in \mathbb{Z} \forall i$. Note that the set $\left\{\gamma\left(K_{i}\right)\right.$ : $1 \leq i \leq n, \gamma \in \Pi\}$ is a finite set of roots of unity; hence the subgroup of $\sqrt{1} \subset R^{\times}$that it generates is cyclic, say $\langle\zeta\rangle$. Thus, $\gamma\left(K_{i}\right)=\zeta^{l_{i}(\gamma)}$ for some $l_{i}: \Pi \rightarrow \mathbb{Z}$.

The above result now says that $\Sigma_{\Pi}(g)=0$ if there exists $\gamma \in \Pi$ so that $|\langle\zeta\rangle|$ does not divide $\sum_{i=1}^{n} n_{i} l_{i}(\gamma)$, and $|\Pi|$ otherwise. (Of course, one can also apply the above result directly to $g=\prod_{i} K_{i}^{n_{i}}$.)

Remark 2.11. From the above proposition, finding out if $g \in G_{\Pi}(H)$ is an important step. However, since $\beta(g)=1 \forall \beta \in[\Pi, \Pi]$, it suffices to compute if $\lambda(g)=1$, where the $\lambda$ 's are the lifts of a set of generators of the (finite) Abelian group $\Pi /[\Pi, \Pi]$. We see more on this in Section 4 below.

\subsection{Application to Quantum Groups and Related Examples}

We now mention some more examples where the above result applies: Hopf algebras that quantize semisimple Lie algebras, their Borel subalgebras, and polynomial algebras (i.e., coordinate rings of affine spaces/Abelian Lie algebras). There are yet other algebras mentioned below, which are not Hopf algebras but can be treated similarly.

To discuss these examples, some more basic results are needed; here is the setup for them. Suppose an $R$-algebra $A$ contains a Hopf subalgebra $H$, so that $A$ is an ad $H$-module (with possible weight spaces $A_{\nu}$ ). Then one has the following result:

Lemma 2.12. Suppose $\mu \in \Gamma_{A}$ (i.e., $\mu: A \rightarrow R$ is an R-algebra map). If $\nu \in \Gamma_{H}$ and $\nu \neq \varepsilon$, then $\mu \equiv 0$ on $A_{\nu}$.

Proof. Given $\nu \neq \varepsilon$, choose $h \in H$ such that $\nu(h) \neq \varepsilon(h)$. Now given $a_{\nu} \in A_{\nu}$, apply $\mu$ to the equation: ad $h\left(a_{\nu}\right)=\nu(h) a_{\nu}$. Simplifying this yields: $\varepsilon(h) \mu\left(a_{\nu}\right)=\nu(h) \mu\left(a_{\nu}\right)$, and since $R$ is an integral domain, $\mu\left(a_{\nu}\right)=0$. 
Applying this easily yields the following result.

Proposition 2.13. ( $A, H$ as above.) Suppose an $R$-algebra $A$ contains $H$ and a vector subspace $V$, that is of the form $V=\oplus_{\nu \neq \varepsilon} V_{\nu}$ (for the ad $H$-action).

1. Every $\mu \in \Gamma_{A}$ kills $A V A$.

2. If $A=H+A V A$, then $\Gamma_{A} \subset \Gamma_{H}$.

3. Say $A=H+A V A$, and $\Pi \subset \Gamma_{A}$ is a finite subgroup of weights of $A$ (from above). If a $\in A$ satisfies $a-\sum_{g \in G(H)} a_{g} g \in A V A$ (where $a_{g} \in R \forall g$ ), then $\Sigma_{\Pi}(a)=|\Pi| \sum_{g \in G_{\Pi}(H)} a_{g}$.

Remark 2.14. Thus, if $\Pi \subset \Gamma_{A}$ is a group (i.e., a subset with a group structure on it), then $\Sigma_{\Pi}(A V A)=0$. Hence, computing $\Sigma_{\Pi}$ at any $a \in A$ essentially reduces to the case of the Hopf subalgebra $H$. When $H$ is a group algebra, Proposition 2.8 above tells us the answer in this case-assuming that the group operation in $\Pi$ agrees with the one in $\Gamma_{H}$.

Moreover, even though $A$ is not a Hopf algebra here, note that the computations come from Hopf algebra calculations (for $H$ ).

Proof. The first part follows from Lemma 2.12, the third part now follows from Proposition 2.8 and Lemma 2.12, and the second part follows by observing that $\mu: H \rightarrow R$ is an algebra map only if $\left.\mu\right|_{G} \in \Gamma_{G}$ and $\left.\mu\right|_{V} \equiv 0$. (Additional relations in $H$ may prevent every $\mu \in \Gamma_{H}$ from being a weight in $\Gamma_{A}$.)

It is now possible to apply the above theory to some examples; note that they are not always Hopf algebras. In each case, $G$ is of the form $\mathfrak{G}_{n, l}$ for some $n, l$. Also choose a special element $q \in R^{\times}$in each case; then $\operatorname{char}(q) \mid l$.

Example 2.15 ("Restricted" quantum groups of semisimple Lie algebras). For this example, $R=k$ is a field with char $k \neq 2$, with a special element $q \neq 0, \pm 1$. Suppose $\mathfrak{g}$ is a semisimple Lie algebra over $k$, together with a fixed Cartan subalgebra and root space decomposition (e.g., using a Chevalley basis, as in [7, Chapter 7]).

One then defines the (Hopf) algebra $U_{q}(\mathfrak{g})$ as in $[8, \S 4.2,4.3]$. In particular, note that it is generated by $\left\{K_{j}^{ \pm 1}, e_{j}, f_{j}: 1 \leq j \leq n\right\}$ (here, $n$ is the rank of $\mathfrak{g}$, and the $\alpha_{i}$ are simple roots), modulo the relations:

$$
K_{i} e_{j} K_{i}^{-1}=q^{\left(\alpha_{i}, \alpha_{j}\right)} e_{j}, K_{i} f_{j} K_{i}^{-1}=q^{-\left(\alpha_{i}, \alpha_{j}\right)} f_{j}, \quad e_{i} f_{j}-f_{j} e_{i}=\delta_{i j} \frac{K_{i}-K_{i}^{-1}}{q_{i}-q_{i}^{-1}}
$$

where $q_{i}=q^{\left(\alpha_{i}, \alpha_{i}\right) / 2}$ for some bilinear form $(\cdot, \cdot)$ on $\mathfrak{h}^{*}$. (We may also need that $q^{4}$ or $q^{6}$ is not 1 , and possibly also that char $k \neq 3$.) The other relations are that $K_{i} K_{j}=K_{j} K_{i}, K_{i}^{ \pm 1} K_{i}^{\mp 1}=1$, and the (two) quantum Serre relations. Define $V:=\oplus_{j=1}^{n}\left(k e_{j} \oplus k f_{j}\right)$.

Now define the "restricted" quantum group as in [9, Chapter 6]. More precisely, given some fixed $l \geq 0$ so that $q^{l}=1$ (whence $\left.\operatorname{char}(q) \mid l\right)$, define the associative (not necessarily Hopf) algebra $u_{q, l}(\mathfrak{g}$ ) to be the quotient of $U_{q}(\mathfrak{g})$ by the relations (for all $j$ ) $K_{j}^{l}=1$, and $e_{j}^{l}=f_{j}^{l}=0$ if $l>0$. Note that $u_{q, l}(\mathfrak{g})=U_{q}(\mathfrak{g})$ if $l=0$, and $u_{q, l}(\mathfrak{g})$ is a Hopf algebra if $l=0$ or $\operatorname{char}(q)$. Moreover, Proposition 2.13 allows us to compute $\Sigma_{\Pi}$ for all $l$.

For each $j$, note that $e_{j}, f_{j}$ are weight vectors (with respect to the adjoint action of the Abelian group $G:=\mathfrak{G}_{n, l}$ generated by all $K_{i}^{ \pm 1}$ ) with weights $q^{ \pm \alpha_{j}} \neq \varepsilon=q^{0}$. Hence Lemma 2.12 implies 
that $\mu\left(e_{j}\right)=\mu\left(f_{j}\right)=0 \forall j, \mu$. Moreover, given the PBW property for $A=U_{q}(\mathfrak{g})$, we know that $A=k \mathfrak{G}_{n, l} \bigoplus\left(V_{-} A+A V_{+}\right)$, where $V_{+}, V_{-}$are the spans of the $e_{j}$ 's and $f_{j}$ 's respectively. Hence $\mu \in \Gamma_{A} \subset \Gamma_{G}$ by the result above.

Every $\mu \in \Gamma_{G}$ is compatible with the commuting of the $K_{i}$ 's, the quantum Serre relations (since $\left.\left.\mu\right|_{V} \equiv 0\right)$, and the " $l^{\text {th }}$ power relations". The only restriction is the last one left, namely: $0=\mu\left(\left[e_{i}, f_{i}\right]\right)$, which gives us that $\mu\left(K_{i}\right)=\mu\left(K_{i}^{-1}\right)$ for all $i, \mu$. Hence $\mu\left(K_{i}\right)= \pm 1$, so that $\Gamma_{A} \cong(\mathbb{Z} / 2 \mathbb{Z})^{n} \cap(\sqrt[l]{1})^{n}$, which is of size $2^{n}$ or 1 , depending on whether $l$ is even or odd. We now compute $\Sigma_{\Pi}(a)$ using the second part of Proposition 2.13, for any $a \in A$.

Example 2.16 (Restricted quantum groups of Borel subalgebras). We consider the subalgebra $A^{\prime}=u_{q, l}(\mathfrak{b})$ of $A=u_{q, l}(\mathfrak{g})$, which is generated by $\left\{K_{i}^{ \pm 1}, e_{i}: 1 \leq i \leq n\right\}$; once again, this algebra quantizes the Borel subalgebra $\mathfrak{b}$ of $\mathfrak{g}$ if $l=0$ (and is a Hopf algebra if $l=0$ or char $(q)$ ). Moreover, every $\mu \in \Gamma_{A^{\prime}}$ kills each $e_{i}$ (where we use $V_{+}$for $V$ ), and we have $\Gamma_{A^{\prime}} \subset \Gamma_{G}$, by Proposition 2.13 above. Moreover, all such maps $\mu \in \Gamma_{G}$ are admissible (i.e., extend to all of $\left.A^{\prime}\right)$, so $\Gamma_{A^{\prime}}=\Gamma_{G} \cong(\sqrt[l]{1})^{n}$.

Now if $\Pi \subset \Gamma_{A^{\prime}}$ is a finite subgroup, then as above, $\Pi \subset \mathfrak{G}_{n, l}^{*}$ (note that $R=k$ here), and furthermore, evaluation of $\Sigma_{\Pi}$ once again reduces to the grouplike case.

Example 2.17 (Taft algebras). Given a primitive $n$th root $q$ of unity, the $n$th Taft algebra is

$$
T_{n}:=R\langle x, g\rangle /\left(g x-q x g, g^{n}-1, x^{n}\right)
$$

Once again, every weight must kill $x$ and sends $g$ to some power of $q$. Hence the set $\Gamma_{T_{n}}$ of weights is cyclic, whence so is $\Pi$. It is now easy to show:

Lemma 2.18. Every weight kills $x$, and $\Sigma_{\Pi}\left(g^{k}\right)=|\Pi|$ if $|\Pi|$ divides $k$, or 0 otherwise.

Example 2.19 (Quantization of affine space). We refer to [10]; once again, $R$ is a unital commutative integral domain. The quantum affine space over $R$ (with a fixed element $q \in R^{\times}$) is the quadratic algebra $T_{R}(V) /\left(x_{j} x_{i}-q x_{i} x_{j}, i<j\right)$, where $V:=\oplus_{i} R x_{i}$. This does not have a Hopf algebra structure; however, Hu presents a quantization of $R[x]:=\operatorname{Sym}_{R}(V)$ in [10, $\left.\S 5\right]$-that is, the quantum group associated to a "finite-dimensional" Abelian Lie algebra. Now consider a more general associative (not necessarily Hopf) $R$-algebra $A=\mathcal{A}_{q, l}(n)$ generated by $\left\{K_{i}^{ \pm 1}, x_{i}: 1 \leq i \leq n\right\}$, with the relations:

$$
\begin{aligned}
& K_{i} K_{j}=K_{j} K_{i}, \quad K_{i}^{l}=1, \quad K_{i}^{ \pm 1} K_{i}^{\mp 1}=1 \\
& K_{i} x_{j} K_{i}^{-1}=\theta_{i j} q^{\delta_{i j}} x_{j}, \quad x_{i} x_{j}=\theta_{i j} x_{j} x_{i}
\end{aligned}
$$

where $\operatorname{char}(q) \mid l$, and $\theta_{i j}$ equals $q$ (respectively $1, q^{-1}$ ) if $i>j$ (respectively $i=j, i<j$ ). Note that $\mathcal{A}_{q, \operatorname{char}(q)}(n)=\mathcal{A}_{q}(n)$, the Hopf algebra introduced and studied by $\mathrm{Hu}$, and $\mathcal{A}_{q, l}(n)$ becomes the Hopf algebra $R[x]$ if $q=l=1$.

We consider the "nontrivial" case $q \neq 1$ (the $q=1$ case is discussed later). Once again, each $x_{j}$ is a weight vector with respect to the (free Abelian) group $G=\mathfrak{G}_{n, l}$, and no $x_{j}$ is in the $\varepsilon$-weight space, so every $\mu \in \Gamma_{A}$ kills $x_{j}$ for all $j$. As in the previous example, $\Gamma_{A} \cong(\sqrt[l]{1})^{n}$, and any finite subgroup $\Pi$ must be contained in $\mathfrak{G}_{n, l}^{*}$.

Moreover, evaluation of $\Sigma_{\Pi}$ once again reduces to the grouplike case. 
Example 2.20 (Quantization of the Virasoro algebra). For this example, assume that $R$ is a field, and $q \in R^{\times}$is not a root of unity. Now refer to [11, Page 100] for the definitions; the Hopf algebra in question is the $R$-algebra $\mathcal{U}_{q}$ generated by $\mathcal{T}, \mathcal{T}^{-1}, c, e_{m}(m \in \mathbb{Z})$ with relations:

$$
\begin{aligned}
\mathcal{T}^{-1} & =\mathcal{T}^{-1} \mathcal{T}=1 \\
q^{2 m} \mathcal{T}^{m} c & =c \mathcal{T}^{m} \\
\mathcal{T}^{m} e_{n} & =q^{-2(n+1) m} e_{n} \mathcal{T}^{m} \\
q^{2 m} e_{m} c & =c e_{m} \\
q^{m-n} e_{m} e_{n}-q^{n-m} e_{n} e_{m} & =[m-n] e_{m+n}+\delta_{m+n, 0} \frac{[m-1][m][m+1]}{[2][3]\langle m\rangle} c
\end{aligned}
$$

where $[m]:=\frac{q^{m}-q^{-m}}{q-q^{-1}}$ and $\langle m\rangle:=q^{m}+q^{-m}$ for all $m \in \mathbb{Z}$.

One can now compute the group of weights, as well as $\Sigma_{\Pi}(h)$ for any monomial word $h$ in the above alphabet. The following is proved using the defining relations above.

Proposition 2.21. Setup as above.

1. The group of weights is $\Gamma_{\mathcal{U}_{q}}=\left(R^{\times}, \cdot\right)$ (so every finite subgroup $\Pi$ is cyclic). A weight $r \in R^{\times}$kills $c$ and all $e_{n}$, and sends $\mathcal{T}$ to $r$.

2. $\Sigma_{\Pi}(h)=0$ if the monomial word $h$ contains $c$ or any $e_{n}$. Moreover, $\Sigma_{\Pi}\left(\mathcal{T}^{m}\right)=0$ unless $|\Pi|$ divides $m$, in which case $\Sigma_{\Pi}\left(\mathcal{T}^{m}\right)=|\Pi|$.

Example 2.22 (Quantum linear groups). For the definitions, we refer to [12, §2]. The quantum general (respectively special) linear group $G L_{q}(n)=R_{q}\left[G L_{n}\right]$ (respectively $S L_{q}(n)=R_{q}\left[S L_{n}\right]$ ) is the localization of the algebra $\mathcal{B}$ (defined presently) at the central quantum determinant

$$
\operatorname{det}_{q}:=\sum_{\pi \in S_{n}}(-q)^{l(\pi)} \prod_{i=1}^{n} u_{i, \pi(i)}
$$

(respectively the quotient of $\mathcal{B}$ by the relation $\operatorname{det}_{q}=1$ ). Here, the algebra $\mathcal{B}=R_{q}[\mathfrak{g l}(n)]$ is generated by $\left\{u_{i j}: 1 \leq i, j \leq n\right\}$, with relations

$$
\begin{aligned}
& u_{i k} u_{i l}=q u_{i l} u_{i k}, \quad u_{i k} u_{j k}=q u_{j k} u_{i k} \\
& u_{i l} u_{j k}=u_{j k} u_{i l}, \quad u_{i k} u_{j l}-u_{j l} u_{i k}=\left(q-q^{-1}\right) u_{i l} u_{j k}
\end{aligned}
$$

where $q \in R^{\times}$, and $i<j, k<l$.

As above, it is possible to compute the group of weights $\Gamma$ for both families of algebras, in the "nontrivial" case $q \neq \pm 1$. In either case, note that $\operatorname{det}_{q} \neq 0$. Given any permutation $\pi \in S_{n}$, suppose there exist $i<j$ such that $\pi(i)>\pi(j)$. Then

$$
\left(q-q^{-1}\right) \mu\left(u_{i, \pi(i)} u_{j, \pi(j)}\right)=\mu\left(\left[u_{i, \pi(j)}, u_{j, \pi(i)}\right]\right)=0
$$

whence $\mu\left(u_{i, \pi(i)} u_{j, \pi(j)}\right)=0$ for all $\mu \in \Gamma$.

The only permutations for which this does not happen is $\left\{\pi \in S_{n}: i<j \Rightarrow \pi(i)<\pi(j)\right\}=\{$ id $\}$. Hence $\mu\left(\operatorname{det}_{q}\right)=\prod_{i} \mu\left(u_{i i}\right) \neq 0$, whence no $u_{i i}$ is killed by any $\mu$. But now for $i<l$,

$$
\mu\left(u_{i i} u_{i l}\right)=q \mu\left(u_{i l} u_{i i}\right), \quad \mu\left(u_{i i} u_{l i}\right)=q \mu\left(u_{l i} u_{i i}\right)
$$


whence $\mu\left(u_{i j}\right)=0$ for all $i \neq j, \mu \in \Gamma$. In particular, since $\Delta\left(u_{i j}\right)=\sum_{k=1}^{n} u_{i k} \otimes u_{k j}$ for (all $i, j$ and) both $G L_{q}(n)$ and $S L_{q}(n)$, hence $\Gamma_{G L} \cong\left(R^{\times}\right)^{n}$, and $\Gamma_{S L} \cong\left(R^{\times}\right)^{n-1}$ (both under coordinate-wise multiplication), since $\mu\left(u_{n n}\right)=\prod_{i=1}^{n-1} \mu\left(u_{i i}\right)^{-1}$ in the latter case.

Finally, computing $\Sigma_{\Pi}$ now reduces to the above results and the first example (of the free group $\left.G=\mathfrak{G}_{n, 0}\right)$. This is because any $h \in G L_{q}(n)$ or $S L_{q}(n)$ can be reduced to a sum of monomial words, and such a word is not killed by any $\mu$ if and only if there is no contribution from any $u_{i l}, i \neq l$.

Example 2.23 (Hopf regular triangular algebras). These were defined in [13] (in the special case $\Gamma=1$ ).

Definition 2.24. An associative $k$-algebra $A$ (over a ground field $k$ ) is a Hopf RTA (or HRTA), if:

1. The multiplication map $B_{-} \otimes_{k} H \otimes_{k} B_{+} \rightarrow A$ is an isomorphism, for some (fixed) associative unital $k$-subalgebras $H, B_{ \pm}$of $A$, and $H$ is, in addition, a commutative Hopf algebra.

2. The set $G:=\operatorname{Hom}_{k-a l g}(H, k)$ contains a free Abelian group with finite basis $\Delta$, so that $B_{ \pm}=\bigoplus_{\lambda \in \pm \mathbb{Z}_{\geqslant 0} \Delta}\left(B_{ \pm}\right)_{\lambda}$. Each summand here is a finite-dimensional weight space for the (usual) adjoint action of $H$, and $\left(B_{ \pm}\right)_{0}=k$.

3. There exists an anti-involution $i$ of $A$, so that $\left.i\right|_{H}=\left.\mathrm{id}\right|_{H}$.

This is a large family of algebras that are widely studied in representation theory. Examples (when $\operatorname{char}(k)=0$ ) are $U(\mathfrak{g})$ for $\mathfrak{g}$ a semisimple, symmetrizable Kac-Moody, centerless Virasoro, or (centerless) extended Heisenberg Lie algebra. Other examples include quantum groups $U_{q}(\mathfrak{g})$ or (quantized) infinitesimal Hecke algebras over $\mathfrak{s l}_{2}$.

In all these examples, the computations reduce to $H$ :

Lemma 2.25. $\Gamma_{A} \subset \Gamma_{H}$.

The same happens if one works with skew group rings over $A$, for example, wreath products $S_{n} \curlywedge A:=A^{\otimes n} \rtimes S_{n}$; in this case $H$ is replaced in the above lemma, by the Hopf subalgebra $H^{\otimes n} \rtimes S_{n}$ of $S_{n} \prec A$.

Proof. Use Proposition 2.13, with $V=N_{-}+N_{+}$, where $N_{ \pm}$are the augmentation ideals in $B_{ \pm}$. Hence $\Gamma_{A} \subset \Gamma_{H}$ (note that each weight must also kill $[A, A] \cap H$ ).

Example 2.26 (Finite-dimensional pointed Hopf algebras). Assume that:

1. $R=\overline{k(R)}$ is an algebraically closed field of characteristic zero.

2. $H$ is a finite-dimensional pointed Hopf algebra over $R$.

3. $G(H)$ is a (finite) Abelian group of order coprime to 210 .

Then by the Classification Theorem 0.1 of [2], $H$ is generated by $G(H)$ and some skew-primitive (defined in the next section) generators $\left\{x_{i}\right\}$ that satisfy $g x_{i} g^{-1}=\chi_{i}(g) x_{i}$ for all $i$ and all $g \in G(H)$. Since $\chi_{i} \neq \varepsilon \forall i$ by [2, Equation (0.1)], hence Proposition 2.13 again reduces the computations here, to the grouplike case: $\Gamma_{H} \subset \Gamma_{G(H)}$. 


\section{Skew-Primitive Elements}

As the previous example 2.26 suggests, large families of Hopf algebras that are the subject of much study in the literature are generated by grouplike and "skew-primitive" elements. Hence in the rest of this paper, we address the question of computing $\Sigma_{\Pi}$ at (monomial words in) such elements.

Definition 3.1. An element $h \in H$ is skew-primitive if $\Delta(h)=g \otimes h+h \otimes g^{\prime}$ for grouplike $g, g^{\prime} \in G(H)$. Denote the set of such elements by $H_{g, g^{\prime}}$. Then $g-g^{\prime} \in H_{g, g^{\prime}} \cap H_{g^{\prime}, g}$, and $H_{1,1}=H_{\text {prim }}$ (recall Definition 2.1).

An element $h$ is pseudo-primitive (respectively almost primitive) with respect to $\Pi$ if, moreover, $g^{-1} g^{\prime} \in G_{\Pi}(H)$ (respectively $g, g^{\prime} \in G_{\Pi}(H)$ ). In future, we may not specify the finite subgroup $\Pi$ of $\Gamma_{H}$, because it is part of the given data.

Thus, $\{$ skew-primitive $\} \supset\{$ pseudo-primitive $\} \supset\{$ almost primitive $\} \supset\{$ primitive $\}$.

Remark 3.2. Note that grouplike and skew-primitive elements in $H$ correspond to 1 -dimensional and non-semisimple 2-dimensional $H$-comodules, respectively. To see this, suppose $V_{0}=R v_{0}$ and $g \in H$. Define $\rho\left(v_{0}\right):=v_{0} \otimes g$. Then $\rho$ induces an $H$-comodule structure on $V_{0}$ if and only if $g$ is grouplike. Similarly, given $V_{1}=R v_{0} \oplus R v_{1}$ and $g, g^{\prime}, h \in H$, define:

$$
\rho\left(v_{0}\right):=v_{0} \otimes g, \quad \rho\left(v_{1}\right)=v_{0} \otimes h+v_{1} \otimes g^{\prime}
$$

Then $\rho$ induces an $H$-comodule structure on $V_{0}$ if and only if $g, g^{\prime}$ are grouplike and $h \in H_{g, g^{\prime}}$ is $\left(g, g^{\prime}\right)$-skew-primitive.

Lemma 3.3. Suppose $\Pi \subset \Gamma_{H}$ is a finite subgroup of weights, and $h \in H_{g, g^{\prime}}$ is as above.

1. The set $\left\{\gamma \in \Gamma_{H}: \gamma(h)=0\right\}$ is a subgroup of $\Gamma_{H}$.

2. $\varepsilon(h)=0$ and $S(h)=-g^{-1} h\left(g^{\prime}\right)^{-1} \in H_{\left(g^{\prime}\right)^{-1}, g^{-1}}$.

3. If $g_{0}$ is any grouplike element, then $g_{0} h$ and $h g_{0}$ are also skew-primitive. Moreover, $g_{0}-g_{0}^{-1} \in$ $H_{g_{0}, g_{0}^{-1}} \cap H_{g_{0}^{-1}, g_{0}}$.

4. For all $n \geq 0$, one also has:

$$
\Delta^{(n)}(h)=\sum_{i=0}^{n} g^{\otimes i} \otimes h \otimes\left(g^{\prime}\right)^{\otimes(n-i)}
$$

5. For any $\gamma \in \Pi$, either $\gamma(g) \neq \gamma\left(g^{\prime}\right)$, or $\gamma(h)=0$, or $\operatorname{char}(R) \mid n_{\gamma}$.

6. $h$ is pseudo-primitive if and only if $g^{-1} h, h g^{-1},\left(g^{\prime}\right)^{-1} h, h\left(g^{\prime}\right)^{-1}$ are almost primitive. If $g_{0} \in G(H)$ and $h$ is pseudo-primitive, then so are $g_{0} h$ and $h g_{0}$.

Proof. The first part is an easy verification. The second part follows from the statements

$$
(\mathrm{id} * \varepsilon)(h):=\mu(\mathrm{id} \otimes \varepsilon) \Delta(h)=h, \quad(\mathrm{id} * S)(h):=\mu(\mathrm{id} \otimes S) \Delta(h)=\varepsilon(h)
$$

The third, fourth and last parts are now easy to verify. For the fifth part, suppose $\gamma(g)=\gamma\left(g^{\prime}\right)$ and $\gamma$ has order $n_{\gamma} \geq 1$ in $\Pi$. Then compute using Equation (3.4) above:

$$
0=\varepsilon(h)=\gamma^{* n_{\gamma}}(h)=n_{\gamma} \gamma(h) \gamma(g)^{n_{\gamma}-1}
$$

The result follows, since $\gamma(g)$ is a unit and $R$ is an integral domain. 
Our main result here is to compute $\Sigma_{\Pi}\left(g_{1} h g_{2}\right)$ for any $g_{1}, g_{2} \in G(H)$, or equivalently by the lemma above, $\Sigma_{\Pi}(h)$ for all skew-primitive $h$. Equation (3.4) implies that if $\gamma \in \Gamma_{H}$, and $\gamma(g) \neq \gamma\left(g^{\prime}\right)$, then

$$
\gamma^{n}(h)=\gamma^{\otimes n}\left(\Delta^{(n-1)}(h)\right)=\gamma(h) \cdot \frac{\gamma(g)^{n}-\gamma\left(g^{\prime}\right)^{n}}{\gamma(g)-\gamma\left(g^{\prime}\right)}
$$

\subsection{The Main Result}

In all that follows below, assume that $h$ is skew-primitive, with $\Delta(h)=g \otimes h+h \otimes g^{\prime}$.

Theorem 3.6. A skew primitive $h \in H_{g, g^{\prime}}$ satisfies at least one of the following three conditions:

1. If there is $\lambda \in \Pi$ so that $\lambda(g), \lambda\left(g^{\prime}\right) \neq 1$, then $\Sigma_{\Pi}(h)=0$. If no such $\lambda$ exists, then one of $g, g^{\prime}$ is in $G_{\Pi}(H)$.

2. Suppose only one of $g, g^{\prime}$ is in $G_{\Pi}(H)$, so that there exists $\lambda \in \Pi$ with exactly one of $\lambda(g), \lambda\left(g^{\prime}\right)$ equal to 1. Then $\Sigma_{\Pi}(h)=\frac{|\Pi| \lambda(h)}{1-\lambda\left(g g^{\prime}\right)}$.

3. If $\lambda\left(g g^{\prime}\right)=1$ for all $\lambda \in \Pi$, then $\Sigma_{\Pi}(h)=\sum_{\gamma \neq \varepsilon=\gamma^{2}} \gamma(h)$, and $2 \Sigma_{\Pi}(h)=0$.

\section{Remark 3.7.}

1. Thus, the expression $\lambda(h) /\left(1-\lambda\left(g g^{\prime}\right)\right)=\Sigma_{\Pi}(h) \in k(R)$ is independent of $\lambda$ (as long as $\lambda\left(g g^{\prime}\right) \neq 1$ ), for such $h$. As the proof indicates, $1-\lambda\left(g g^{\prime}\right)$ should really be thought of as $1-\lambda(g)$ or $1-\lambda\left(g^{\prime}\right)$ (depending on which of $g^{\prime}$ and $g$ is in $G_{\Pi}(H)$ ).

Moreover, Equation (3.5) implies, whenever $\gamma^{n}(g) \neq \gamma^{n}\left(g^{\prime}\right)$, that

$$
\frac{\gamma^{n}(h)}{\gamma^{n}(g)-\gamma^{n}\left(g^{\prime}\right)}=\frac{\gamma(h)}{\gamma(g)-\gamma\left(g^{\prime}\right)}
$$

It is not hard to show that both of these are manifestations of the following easy fact:

Lemma 3.8. Given $h \in H_{g, g^{\prime}}$, define $N_{h}:=\left\{\gamma \in H^{*}: \gamma(g) \neq \gamma\left(g^{\prime}\right)\right\}$. Suppose $\mu, \lambda \in N_{h}$. Then $(\mu * \lambda)(h)=(\lambda * \mu)(h)$ if and only if $f_{h}(\mu)=f_{h}(\lambda)$, where $f_{h}: N_{h} \rightarrow k(R)$ is given by

$$
f_{h}(\gamma):=\frac{\gamma(h)}{\gamma(g)-\gamma\left(g^{\prime}\right)}
$$

(In other words, weights commute at $h$ precisely when they lie on the same "level surface" for $f_{h}$.)

2. Also note that if the first two parts fail to hold, then both $g, g^{\prime} \in G_{\Pi}(H)$, and the final part holds. Thus, the above theorem computes $\Sigma_{\Pi}(h)$ for all skew-primitive $h$, if $\operatorname{char}(R) \neq 2$ or not both of $g, g^{\prime}$ are in $G_{\Pi}(H)$. We address the case when $\operatorname{char}(R)=2$ and $g, g^{\prime} \in G_{\Pi}(H)$, in the next subsection.

\section{Proof of Theorem 3.6.}

1. Apply Equation (2.4) to $h$, to get $\Sigma_{\Pi}(h)=\lambda(g) \Sigma_{\Pi}(h)+\lambda(h) \Sigma_{\Pi}\left(g^{\prime}\right)$. Since $g^{\prime} \notin G_{\Pi}(H)$, hence the second term vanishes, and we are left with $(1-\lambda(g)) \Sigma_{\Pi}(h)=0$. But $\lambda(g) \neq 1$.

Next, if no such $\lambda \in \Pi$ exists, then $\Pi=\Pi_{g} \cup \Pi_{g^{\prime}}$, where $\Pi_{g}, \Pi_{g^{\prime}}$ were defined before Proposition 2.8. We claim that one of the two sets is contained in the other whence one of $g, g^{\prime}$ is in $G_{\Pi}(H)$, because, if not, then one can choose $\gamma, \gamma^{\prime} \in \Pi$ so that neither $\gamma(g)$ nor $\gamma^{\prime}\left(g^{\prime}\right)$ equals 1 (whence $\left.\gamma\left(g^{\prime}\right)=1=\gamma^{\prime}(g)\right)$. Then one verifies that $\gamma \gamma^{\prime} \notin \Pi_{g} \cup \Pi_{g^{\prime}}$, which is a contradiction. Thus one of $\Pi_{g} \backslash \Pi_{g^{\prime}}, \Pi_{g^{\prime}} \backslash \Pi_{g}$ is empty. 
2. Suppose $g^{\prime} \in G_{\Pi}(H), \lambda(g) \neq 1$ for some $\lambda$ (the other case is similar). Now apply Equation (2.4) and Proposition 2.8, and compute:

$$
\Sigma_{\Pi}(h)=\lambda(h) \frac{\Sigma_{\Pi}(g)-\Sigma_{\Pi}\left(g^{\prime}\right)}{\lambda(g)-\lambda\left(g^{\prime}\right)}=\lambda(h) \frac{0-|\Pi|}{\lambda(g) \cdot 1-1}=\frac{\lambda(h)|\Pi|}{1-\lambda(g) \lambda\left(g^{\prime}\right)}
$$

3. If $\lambda\left(g g^{\prime}\right)=1$ for any $\lambda \in \Pi$, then: $\lambda^{-1}(h)=-\lambda(h)$. Thus $\lambda+\lambda^{-1}$ kills $h$, and the first equation now follows because $\varepsilon(h)=0$. The second is also easy: $2 \Sigma_{\Pi}(h)=\sum_{\gamma \in \Pi}\left(\gamma(h)+\gamma^{-1}(h)\right)=0$.

\subsection{The Characteristic 2 Case}

The only case that Theorem 3.6 does not address is when $\operatorname{char}(R)=2$ and $g, g^{\prime} \in G_{\Pi}(H)$. We now address this case.

Proposition 3.9. Suppose $\Pi$ is as above, $\operatorname{char}(R)=2$, and $h \in H_{g, g^{\prime}}$ is almost primitive with respect to $\Pi$.

1. If $\Pi$ has odd order, then $\Sigma_{\Pi}(h)=0$.

2. If 4 divides $|\Pi|$, then $\Sigma_{\Pi}(h)=0$.

3. If $\Pi$ has even order but $4 \nmid|\Pi|$, then $\Sigma_{\Pi}(h)=\gamma(h)$ for any $\gamma \in \Pi$ of order exactly 2. This may assume any nonzero value in $R$.

We omit the proof, since this result is a special case of more general results in general (positive) characteristic, which we state and prove later. See Theorems 7.8 and 7.10, as well as Remark 7.11.

\subsection{A Degenerate Example: Quiver (Co)algebras}

We conclude this section with an example that is not a Hopf algebra, but an algebra with coproduct, and is generated by grouplike and skew-primitive elements. (Thus, $\Pi$ is no longer necessarily a group of weights, but a semigroup.)

Consider a quiver $Q=\left(Q_{0}, Q_{1}\right)$, which is a directed graph with vertex set $Q_{0}$ and edges $Q_{1}$. Thus, there exist source and target maps $s, t: Q_{1} \rightarrow Q_{0}$ such that every edge $e \in Q_{1}$ starts at $s(e)$ and ends at $t(e)$. A path in $Q$ is a finite sequence of edges $a_{1} \cdots a_{n}$ such that $t\left(a_{i}\right)=s\left(a_{i+1}\right)$. We also write $s(p):=s\left(a_{1}\right)$ and $t(p):=t\left(a_{n}\right)$, and the length of the path is said to be $n$. Vertices $v \in Q_{0}$ are paths of length zero, and one writes $s(v)=t(v):=v$.

There are two structures on the free $R$-module $R Q$ with basis consisting of all paths in $Q$. The path algebra is defined by setting the product of two paths $a_{1} \cdots a_{n}$ and $b_{1} \cdots b_{m}$ to be their concatenation $a_{1} \cdots a_{n} b_{1} \cdots b_{m}$ if $t\left(a_{n}\right)=s\left(b_{m}\right)$; otherwise the product is zero. Then $R Q$ is an associative $R$-algebra that contains enough idempotents $\left\{v: v \in Q_{0}\right\}$. However, $R Q$ contains a unit if and only if $Q_{0}$ is finite, in which case the unit is $\sum_{v \in Q_{0}} v$.

Another structure on $R Q$ is given by defining $\Delta: R Q \rightarrow R Q \otimes R Q$ via: $\Delta(p):=\sum_{(q, r): q r=p} q \otimes r$. Also define $\varepsilon: R Q \rightarrow R$ via: $\varepsilon(v):=1$ for all $v \in Q_{0}$ and $\varepsilon(p):=0$ for all paths $p$ of positive length. This structure makes $R Q$ into the path coalgebra. 
The path (co)algebra has been the subject of much study in the literature, and it is natural to ask for which quivers $Q$ are these two structures on $R Q$ compatible. It is not hard to show that the answer is: very few.

Lemma 3.10. The coproduct $\Delta$ is multiplicative if and only if there are no paths of length $\geq 2$.

Thus, the quiver bialgebra is a "degenerate" example. Now suppose $Q$ has no paths of length $\geq 2$ (and hence, no self-loops). Then $\Delta$ is indeed multiplicative. Nevertheless, if $1<\left|Q_{0}\right|<\infty$, then the unit in $R Q$ is not grouplike. It is now possible to show the following.

Proposition 3.11. There exists a bijection from $Q_{0} \amalg\{0\}$ to the semigroup $\Gamma_{R Q}$, sending $v$ to $\lambda_{v}$ that sends $v$ to 1 and all other paths to 0 .

Proof. Given $v \neq v^{\prime} \in Q_{0}$ and $\lambda \in \Gamma_{R Q}$,

$$
\lambda(v)=\lambda\left(v^{2}\right)=\lambda(v)^{2}, \quad \lambda(v) \lambda\left(v^{\prime}\right)=\lambda\left(v v^{\prime}\right)=0
$$

Since $R$ is a unital integral domain, this implies that at most one $\lambda(v)$ is nonzero-and then it equals 1 . Moreover, if $p$ is any path of length 1 in $Q_{1}$, then at least one of its vertices is killed by $\lambda$, whence so is $p$. This supplies the desired bijection. In particular, $\Gamma_{R Q} \cong Q_{0} \amalg\{0\}$ as semigroups, with composition given by:

$$
\lambda_{v} * \lambda_{v^{\prime}}=\delta_{v, v^{\prime}} \lambda_{v}, \quad \lambda_{v} * 0=0 * \lambda_{v^{\prime}}=0, \quad \forall v, v^{\prime} \in Q_{0}
$$

In fact, the path algebra and path coalgebra are dual to one another, morally speaking. More precisely, each of them can be "recovered" inside the dual space of the other; see [14] for more details.

\section{Subgroups and Subquotients of Groups of Weights}

\subsection{Subgroups Associated to Arbitrary Elements}

Recall that the goal of this article is to compute $\Sigma_{\Pi}$ at any element $h$ in a Hopf algebra $H$. We start this section with the following constructions.

Definition 4.1. Suppose $R$ is a commutative unital integral domain.

1. Suppose $H$ is an $R$-algebra, such that $\Gamma=\Gamma_{H}$ has a group structure $*$ on it. Define $\Gamma_{h} \subset \Gamma$ to be the subgroup of $\Gamma$ that "stabilizes" $h$. In other words,

$$
\Gamma_{h}:=\{\gamma \in \Gamma:(\beta * \gamma * \delta)(h)=(\beta * \delta)(h) \forall \beta, \delta \in \Gamma\}
$$

2. Given a coalgebra $H$, and $h \in H$, define $C_{h}$ to be the $R$-subcoalgebra generated by $h$ in $H$.

3. Given a Hopf algebra $H$, define $\Gamma_{h}^{\prime}$ to be the fixed weight monoid of $h$, given by $\Gamma_{h}^{\prime}:=\{\gamma \in \Gamma$ : $\left.\left.\gamma\right|_{C_{h}}=\left.\varepsilon\right|_{C_{h}}\right\}$.

In particular, $\gamma(h)=\varepsilon(h)$ if $\gamma \in \Gamma_{h}$.

A later subsection will discuss how this allows us to consider subquotients of $\Gamma$; but first, here are some observations involving these subgroups. 
Proposition 4.2. Suppose $H$ is a Hopf algebra, and $h \in H$.

1. For all $h, \Gamma_{h}$ is a normal subgroup of $\Gamma$, and $\Gamma_{h}^{\prime} \subset \Gamma_{h}$ is a monoid closed under $\Gamma$-conjugation.

2. Given $\left\{h_{i}: i \in I\right\} \subset H$, and $h \in\left\langle h_{i}\right\rangle$ (i.e., in the subalgebra generated by the $h_{i}$ 's), $\Gamma_{h} \supset$ $\bigcap_{i \in I} \Gamma_{h_{i}}$, and similarly for the $\Gamma^{\prime} s$.

3. Given any $h_{i} \in H$ (finitely many), suppose $\Pi=\times_{i} \Pi_{i}$, with $\Pi_{i} \subset \Gamma_{h_{j}}^{\prime}$ whenever $i \neq j$. Then $\Sigma_{\Pi}(\mathbf{h})=\prod_{i} \Sigma_{\Pi_{i}}\left(h_{i}\right)$

Proof. The first and third parts are straightforward computations. For the second part, for all $\beta$, $\delta \in \Gamma, \gamma \in \bigcap_{i} \Gamma_{h_{i}}$, and polynomials $p$ in the $h_{i}$ 's,

$$
(\beta * \gamma * \delta)\left(p\left(h_{i}\right)\right)=p\left((\beta * \gamma * \delta)\left(h_{i}\right)\right)=p\left((\beta * \delta)\left(h_{i}\right)\right)=(\beta * \delta)\left(p\left(h_{i}\right)\right)
$$

The outer equalities hold because weights are algebra maps.

The proof for the $\Gamma^{\prime}$ s is as follows: if $h=p\left(h_{i}\right)$ as above, then since $\Delta$ is multiplicative, hence any $h^{\prime} \in C_{h}$ is expressible as a polynomial in elements $h_{j}^{\prime} \in \cup_{i} C_{h_{i}}$ - say $h^{\prime}=q\left(h_{j}^{\prime}\right)$. In particular, if $\gamma \in \Gamma_{h_{i}}^{\prime}$ for all $i$, then

$$
\gamma\left(q\left(h_{j}^{\prime}\right)\right)=q\left(\gamma\left(h_{j}^{\prime}\right)\right)=q\left(\varepsilon\left(h_{j}^{\prime}\right)\right)=\varepsilon\left(q\left(h_{j}^{\prime}\right)\right)
$$

where once again, the outer equalities hold because weights are algebra maps. In other words, $\gamma\left(h^{\prime}\right)=\varepsilon\left(h^{\prime}\right)$.

We also mention two examples; the proofs are straightforward.

Lemma 4.3. If $g \in G(H)$, then this definition of $\Gamma_{g}$ coincides with the previous one: $\Gamma_{g}=\Gamma_{g}^{\prime}=\{\gamma \in$ $\Gamma: \gamma(g)=1\}$. If $h \in H_{g, g^{\prime}}$, then $\Gamma_{h} \subset \Gamma_{g} \cap \Gamma_{g^{\prime}}$, or $\Gamma_{h}=\Gamma$. In both cases, $\Gamma_{h}^{\prime}=\Gamma_{h} \cap \Gamma_{g} \cap \Gamma_{g^{\prime}}$.

\subsection{Subquotients}

We now compute $\Sigma_{\Pi}(h)$ for more general $\Pi$. The following result is used later.

Lemma 4.4. Fix $h \in H$, and choose any subgroup $\Gamma^{\prime}$ of $\Gamma_{h}$ that is normal in $\Gamma$. Also fix a finite subgroup $\Pi$ of $\Gamma / \Gamma^{\prime}$. Now fix any lift $\widetilde{\Pi}$ of $\Pi$ to $\Gamma$, and define

$$
\Sigma_{\Pi}(h):=\sum_{\gamma^{\prime \prime} \in \widetilde{\Pi}} \gamma^{\prime \prime}(h), \quad \Pi^{\circ}:=\left\{\gamma \in \Gamma:\left(\gamma+\Gamma^{\prime}\right) \in \Pi \subset \Gamma / \Gamma^{\prime}\right\}
$$

1. $\Sigma_{\Pi}(h)$ is well-defined.

2. If a subgroup $\Gamma^{\prime \prime} \subset \Gamma_{h}$ is normal in $\Pi^{\circ}$ (e.g., $\Gamma^{\prime \prime}=\Gamma^{\prime}, \Gamma_{h} \cap \Pi^{\circ}$ ), then

$$
\Sigma_{\Pi^{\circ}}(h)=\left|\Gamma^{\prime \prime}\right| \Sigma_{\Pi^{\circ} / \Gamma^{\prime \prime}}(h)
$$

Proof. For the first part, choose any other lift $\Pi^{\prime}$ of $\Pi$. If $\gamma^{\prime} \in \Pi^{\prime}, \gamma^{\prime \prime} \in \widetilde{\Pi}$ are lifts of $\gamma \in \Pi$, then $\left(\gamma^{\prime}\right)^{-1} * \gamma^{\prime \prime} \in \Gamma_{h}$, so

$$
\sum_{\gamma^{\prime \prime} \in \widetilde{\Pi}} \gamma^{\prime \prime}(h)=\sum_{\gamma^{\prime} \in \Pi^{\prime}}\left(\gamma^{\prime} *\left(\left(\gamma^{\prime}\right)^{-1} * \gamma^{\prime \prime}\right)\right)(h)=\sum_{\gamma^{\prime} \in \Pi^{\prime}} \gamma^{\prime}(h)
$$


by definition of $\Gamma_{h}$. The other part is also easy, since by the first part, $\Sigma_{\Pi^{*}}(h)$ is also well-defined, where the finite group is $\Pi^{*}:=\Pi^{\circ} / \Gamma^{\prime \prime}$. If $\Pi^{* *}$ is any lift to $\Pi^{\circ}$ of $\Pi^{*}$, then

$$
\Sigma_{\Pi^{\circ}}(h)=\sum_{\gamma^{\prime} \in \Pi^{* *}, \beta \in \Gamma^{\prime \prime}}\left(\gamma^{\prime} * \beta\right)(h)=\sum_{\gamma^{\prime} \in \Pi^{* *}, \beta \in \Gamma^{\prime \prime}} \gamma^{\prime}(h)=\left|\Gamma^{\prime \prime}\right| \sum_{\gamma^{\prime} \in \Pi^{* *}} \gamma^{\prime}(h)
$$

and this equals the desired amount.

While a special case of the second part is that $\Sigma_{\Pi^{\circ}}(h)=\left|\Gamma^{\prime}\right| \Sigma_{\Pi}(h)$, we really use the result when $\Gamma^{\prime}$ is itself finite, and we replace $\Pi^{\circ}$ by $\Pi$. The equation is then used to compute $\Sigma_{\Pi}(h)$.

\subsection{Pseudo-Primitive Elements}

For the rest of this paper, $H$ is an $R$-Hopf algebra, unless stated otherwise. If $h$ is grouplike or (pseudo-)primitive, then it is easy to see that $\Sigma_{\Pi}(h)=|[\Pi, \Pi]| \Sigma_{\Pi_{a b}}(h)$, where $\Pi_{a b}:=\Pi /[\Pi, \Pi]$ (we show the pseudo-primitive case presently). Thus, in the grouplike case, the question of whether or not $h \in G_{\Pi}(H)$ reduces to evaluating (any lift of a set of) generators of the finite Abelian group $\Pi_{a b}$, at $h$.

Proposition 4.5. Suppose $h \in H_{g, g^{\prime}}$ is pseudo-primitive with respect to $\Pi$.

1. Then $(\gamma * \nu)(h)=(\nu * \gamma)(h)=\nu(g) \gamma(h)+\gamma(g) \nu(h)$ for all $\gamma, \nu \in \Pi$.

2. $\Gamma_{h}^{\prime} \supset[\Pi, \Pi]$.

3. For any $m \geq 0, \gamma^{* m}(h)=m \gamma(g)^{m-1} \gamma(h)$ if $\gamma \in \Pi$. In particular, if char $(R)=p$ is prime, then $\gamma^{* p}(h)=0 \forall \gamma \in \Pi$.

Proof. The first and last parts are by definition and induction respectively. As for the second part, one shows the following computation for any skew-primitive $h \in H_{g, g^{\prime}}$, and $\beta, \beta^{\prime} \in \Gamma_{H}$ :

$$
\begin{aligned}
\left(\beta * \beta^{\prime} * \beta^{-1} *\left(\beta^{\prime}\right)^{-1}\right)(h)= & \beta\left(h\left(g^{\prime}\right)^{-1}\right)\left(1-\beta^{\prime}\left(g\left(g^{\prime}\right)^{-1}\right)\right) \\
& +\beta^{\prime}\left(h\left(g^{\prime}\right)^{-1}\right)\left(\beta\left(g\left(g^{\prime}\right)^{-1}\right)-1\right)
\end{aligned}
$$

using Lemma 3.3. Since $h$ is pseudo-primitive with respect to $\Pi$, this shows that every generator (and hence element) $\lambda$ of $[\Pi, \Pi]$ satisfies: $\lambda(g)=\lambda\left(g^{\prime}\right)=1$ and $\lambda(h)=0$. Since $C_{h}=R h+R g+R g^{\prime}$, these imply that $\lambda \in \Gamma_{h}^{\prime}$.

An easy consequence of Propositions 4.2 and 4.5 is

Corollary 4.6. If $h \in H$ is (in the subalgebra) generated by grouplike and pseudo-primitive elements (with respect to $\Pi$ ), then $\Gamma_{h}^{\prime} \supset[\Pi, \Pi]$.

Also note that given some $h \in H$, one can compute $\Sigma_{\Pi}(h)$ for more general $\Pi$, and hence the results in this paper can be generalized; however, we stay with the original setup when $\Pi \subset \Gamma$ (i.e., $\Gamma^{\prime}=\{\varepsilon\}$ ). This (general) case is noteworthy, however, because it is used below.

We conclude by specifying more precisely what is meant by $\Sigma_{\Pi}\left(h_{1} \ldots h_{n}\right)$ for "pseudo-primitive" $h_{i}$ 's, when $\Pi$ is a subquotient of $\Gamma$ as above. In this case, start with some skew-primitive $h_{i}$ 's, then let $\Pi$ be a finite subgroup of $\Gamma / \Gamma^{\prime}$, for some subgroup $\Gamma^{\prime} \subset \cap_{i} \Gamma_{h_{i}}$ that is normal in $\Gamma$. Moreover, if 
$\Delta\left(h_{i}\right)=g_{i} \otimes h_{i}+h_{i} \otimes g_{i}^{\prime}$, then assume further that $\gamma\left(g_{i}\right)=\gamma\left(g_{i}^{\prime}\right)$, for all elements $\gamma$ of the subgroup $\Pi^{\circ}$ (defined above).

This is what is meant in the case of general $\Pi$, when we say that $g_{i}, g_{i}^{\prime} \in G_{\Pi}(H)$-i.e., that the $h_{i}$ 's are pseudo-primitive (with respect to $\Pi$ ). Similarly, to say that the $h_{i}$ 's are almost primitive with respect to $\Pi$ means that $\gamma\left(g_{i}\right)=\gamma\left(g_{i}^{\prime}\right)=1 \forall \gamma \in \Pi^{\circ}$.

\section{Products of Skew-Primitive Elements}

We now mention some results on (finite) products of skew-primitive elements and grouplike elements. From now on, $\Pi$ denotes a finite subgroup of $\Gamma=\Gamma_{H}$ and not a general subquotient; however, in the next section, we need to use a subquotient $\Phi$ of this $\Pi$.

Since the set of skew-primitive elements is closed under multiplication by grouplike elements, any "monomial" in them can be expressed in the form $\mathbf{h}=\prod_{i} h_{i}$. The related "grouplike" elements that would figure in the computations are $\mathbf{g}=\prod_{i} g_{i}$ and $\mathbf{g}^{\prime}=\prod_{i} g_{i}^{\prime}$.

Standing Assumption 5.1. For this section and the next two, assume that $h_{i} \in H_{g_{i}, g_{i}^{\prime}}$ for all (finitely many) $i$.

First, here are some results that hold in general.

Proposition 5.2. If $\lambda\left(h_{i}\right)=0 \forall i$ for some $\lambda \in \Pi$, and (at least) one of $\lambda(\mathbf{g}), \lambda\left(\mathbf{g}^{\prime}\right)$ is not 1 , then $\Sigma_{\Pi}(\mathbf{h})=0$.

Proof. If $\lambda\left(h_{i}\right)=0$ then $\lambda^{m}\left(h_{i}\right)=0$ for all $i, m$. Now choose a set $\mathcal{B}$ of coset representatives for $\langle\lambda\rangle$ in $\Pi$, and assume that $\lambda\left(\mathbf{g}^{\prime}\right) \neq 1$ (the other case is similar). Then compute:

$$
\Sigma_{\Pi}(\mathbf{h})=\sum_{\beta \in \mathcal{B}, \gamma \in\langle\lambda\rangle} \prod_{i}\left(\beta\left(g_{i}\right) \gamma\left(h_{i}\right)+\beta\left(h_{i}\right) \gamma\left(g_{i}^{\prime}\right)\right)
$$

Since $\gamma\left(h_{i}\right)=0$ for all $\gamma \in\langle\lambda\rangle$ and all $i$, hence the entire product in the summand collapses, to give $\sum_{\beta \in \mathcal{B}} \beta(\mathbf{h}) \cdot \Sigma_{\langle\lambda\rangle}\left(\mathbf{g}^{\prime}\right)$. But now the second factor vanishes by our assumption (and Proposition 2.8).

Next, if $\Sigma_{\Pi}\left(\prod_{i=1}^{n} h_{i}\right)$ is known for all skew-primitive $h_{i}$ 's, then one can evaluate the product of $(n+1)$ such $h$ 's in some cases. The following result relates $\Sigma_{\Pi}$-values of strings to the $\Sigma_{\Pi}$-values of proper substrings (with skew-primitive "letters"), which are "corrected" by grouplike elements. The proof is that both equations below follow by evaluating Equation (2.4) at $\mathbf{h}$.

Proposition 5.3. Suppose one of $\mathbf{g}, \mathbf{g}^{\prime}$ is not in $G_{\Pi}(H)$. Thus, if $\lambda(\mathbf{g}) \neq 1$ (or respectively $\lambda\left(\mathbf{g}^{\prime}\right) \neq 1$ ) for some $\lambda \in \Pi$, then

$$
\Sigma_{\Pi}(\mathbf{h})=\frac{\sum_{\nu \in \Pi} \prod_{i}\left(\lambda\left(g_{i}\right) \nu\left(h_{i}\right)+\lambda\left(h_{i}\right) \nu\left(g_{i}^{\prime}\right)\right)-\lambda(\mathbf{g}) \Sigma_{\Pi}(\mathbf{h})}{1-\lambda(\mathbf{g})}
$$

or respectively,

$$
\Sigma_{\Pi}(\mathbf{h})=\frac{\sum_{\nu \in \Pi} \prod_{i}\left(\nu\left(g_{i}\right) \lambda\left(h_{i}\right)+\nu\left(h_{i}\right) \lambda\left(g_{i}^{\prime}\right)\right)-\Sigma_{\Pi}(\mathbf{h}) \lambda\left(\mathbf{g}^{\prime}\right)}{1-\lambda\left(\mathbf{g}^{\prime}\right)}
$$


Note here that both numerators on the right side have an $\Sigma_{\Pi}(\mathbf{h})$ in them, which cancels the only such term present in the summations. Thus, what one is left with in either case are linear combinations of $\Sigma_{\Pi}$-values of "corrected" proper substrings, with coefficients of the form $\lambda\left(\prod g_{j} h_{k}\right)$.

Also note that if the $\Sigma_{\Pi}$-values of all "corrected" proper substrings are known, and $\operatorname{char}(R) \neq 2$, then the two propositions, one above and one below, can be used, for instance, to compute $\Sigma_{\Pi}$ at all monomials of odd length (in skew-primitive elements).

The statement and proof of the following result are essentially the same as those of the last part of Theorem 3.6 above.

Proposition 5.4. Suppose $\mathrm{gg}^{\prime} \in G_{\Pi}(H)$, and $\operatorname{char}(R)=2$ if the number of $h_{i}$ 's is even. Then $\Sigma_{\Pi}(\mathbf{h})=\sum_{\gamma \neq \varepsilon=\gamma^{2}} \gamma(\mathbf{h})$, and $2 \Sigma_{\Pi}(\mathbf{h})=0$.

This is because once again, one shows that $\lambda^{-1}(\mathbf{h})=-\lambda(\mathbf{h}) \forall \lambda$.

The next result in this subsection is true for almost all values of char $R$. The proof is immediate from the penultimate part of Lemma 3.3 above.

Proposition 5.5. If $\operatorname{char}(R) \nmid|\Pi|$ and $g_{i}^{-1} g_{i}^{\prime} \in G_{\Pi}(H)$ (i.e., $h_{i}$ is pseudo-primitive) for some $i$, then $\gamma\left(h_{i}\right)=0 \forall \gamma \in \Pi$. In particular, $\Sigma_{\Pi}(\mathbf{h})=0$.

We conclude this section with one last result—in characteristic $p$.

Theorem 5.6. Suppose $\operatorname{char}(R)=p>0$, and $h_{i} \in H_{g_{i}, g_{i}^{\prime}}$ for all $i$. Choose and fix a $p$-Sylow subgroup $\Pi_{p}$ of $\Pi$.

1. Then each $h_{i}$ is almost primitive with respect to $\Pi_{p}$.

2. If $\Pi_{p}$ contains an element of order $p^{2}$, then $\Sigma_{\Pi}(\mathbf{h})=\Sigma_{\Pi_{p}}(\mathbf{h})=0$.

It is also shown later that $\Sigma_{\Pi_{p}}(\mathbf{h})=0$ whenever $\Pi_{p} \not(\mathbb{Z} / p \mathbb{Z})^{k}$ for any $k>0$.

Proof.

1. If $z^{p}=1$ in $R$, then $(1-z)^{p}=1-z^{p} \bmod p=0$ in $R$. Since $R$ is an integral domain, $z=1$. Now assume that $\left|\Pi_{p}\right|=p^{f}$. Then $\gamma^{* p^{f}}=\varepsilon$ for each $\gamma \in \Pi_{p}$, whence $\gamma\left(g_{i}\right)^{p^{f}}=\gamma\left(g_{i}^{\prime}\right)^{p^{f}}=1$ for all $i$. Successively set $z=\gamma\left(g_{i}\right)^{p^{t}}$, for $t=f-1, f-2, \ldots, 1,0$. Hence $\gamma\left(g_{i}\right)=1$ for all $i$; the other case is the same.

2. Next, if $\lambda$ has order $p^{2}$, then choose a set $\mathcal{B}$ of coset representatives for $\langle\lambda\rangle$ in $\Pi$, and compute using Proposition 4.5 above (since all $h_{i}$ 's are pseudo/almost primitive with respect to $\Pi_{p}$, hence for $\langle\lambda\rangle)$ :

$$
\Sigma_{\Pi}(\mathbf{h})=\sum_{\beta \in \mathcal{B}} \sum_{j=0}^{p^{2}-1} \prod_{i=1}^{n}\left(\beta * \lambda^{* j}\right)\left(h_{i}\right)=\sum_{\beta \in \mathcal{B}} \sum_{j=0}^{p^{2}-1} \prod_{i=1}^{n}\left(\beta\left(h_{i}\right) \lambda^{j}\left(g_{i}\right)+j \beta\left(g_{i}\right) \lambda\left(g_{i}\right)^{j-1} \lambda\left(h_{i}\right)\right)
$$

Call the factor in the product $a_{i, j}$ (it really is $a_{\beta, i, j}$ ). Now observe that $a_{i, p+j}=\lambda^{p}\left(g_{i}\right) a_{i, j}$ for all $i, j$, whence $a_{i, k p+j}=\lambda^{k p}\left(g_{i}\right) a_{i, j}$. Therefore for any $\beta \in \mathcal{B}$, one can take $\sum_{k=0}^{p-1} \lambda(\mathbf{g})^{k p}$ out of the summand. But $\lambda(\mathbf{g})^{p}=1$, so every $\beta$-summand vanishes. 


\section{Special Case: Abelian Group of Weights}

In this section, the focus is on evaluating $\Sigma_{\Pi}(\mathbf{h})$ in the special case where the group $\Pi$ of weights is Abelian.

\section{Definition 6.1.}

1. For all $n \in \mathbb{N}$, define $[n]:=\{1,2, \ldots, n\}$.

2. Given $I \subset[n]$, define $g_{I}:=\prod_{i \in I} g_{i}$, and similarly define $g_{I}^{\prime}, h_{I}$.

3. Define $\Pi_{p}$ to be any fixed $p$-Sylow subgroup of $\Pi$ if $\operatorname{char}(R)=p>0$, and $\{\varepsilon\}$ otherwise. Also choose and fix a "complementary" subgroup $\Pi^{\prime}$ to $\Pi_{p}$ in $\Pi$ (if $\Pi$ is Abelian), i.e., $\left|\Pi_{p}\right| \cdot\left|\Pi^{\prime}\right|=|\Pi|$. (And if $\operatorname{char}(R)=0$, set $\Pi^{\prime}:=\Pi$.)

We now present two results. The first is (nontrivial only) when $\operatorname{char}(R)$ divides the order of $\Pi$, and the second (which really is the main result) is when it does not.

Theorem 6.2. Suppose $\Pi$ is Abelian; let $\Pi_{p}, \Pi^{\prime}$ be as above. Let $J \subset[n]$ be the set of $i$ 's such that $h_{i}$ is pseudo-primitive with respect to $\Pi$. Then

$$
\Sigma_{\Pi}(\mathbf{h})=\Sigma_{\Pi^{\prime}}\left(g_{J} h_{[n] \backslash J}\right) \cdot \Sigma_{\Pi_{p}}\left(h_{J}\right)
$$

In particular, if $\operatorname{char}(R)=0$, or $0<\operatorname{char}(R) \nmid|\Pi|$, then $\Sigma_{\Pi_{p}}\left(h_{J}\right)=\varepsilon\left(h_{J}\right)=0$, whence $\Sigma_{\Pi}(\mathbf{h})=0$ too. Proof. First note that $\lambda\left(g_{i}\right)=\lambda\left(g_{i}^{\prime}\right)=1$ for all $\lambda \in \Pi_{p}$ and all $i$, by Theorem 5.6 above. Since $\Pi$ is Abelian, every $\gamma \in \Pi$ is uniquely expressible as $\gamma=\beta * \lambda$ with $\beta \in \Pi^{\prime}, \lambda \in \Pi_{p}$. We now compute $\gamma\left(h_{i}\right)$ in both cases: $i \in J$ and $i \notin J$.

First consider the case when $i \in J$. Then $\beta\left(h_{i}\right)=0$ for all $\beta \in \Pi^{\prime}$, by Proposition 5.5. Thus, $\gamma\left(h_{i}\right)=\beta\left(g_{i}\right) \lambda\left(h_{i}\right)+\beta\left(h_{i}\right) \lambda\left(g_{i}\right)=\beta\left(g_{i}\right) \lambda\left(h_{i}\right)$.

Now suppose $i \notin J$. Choose $\gamma \in \Pi$ so that $\gamma\left(g_{i}\right) \neq \gamma\left(g_{i}^{\prime}\right)$. Then $(\gamma * \lambda)\left(h_{i}\right)=(\lambda * \gamma)\left(h_{i}\right)$, which leads (upon simplifying) to

$$
\lambda\left(h_{i}\right)\left(\gamma\left(g_{i}\right)-\gamma\left(g_{i}^{\prime}\right)\right)=\gamma\left(h_{i}\right)\left(\lambda\left(g_{i}\right)-\lambda\left(g_{i}^{\prime}\right)\right)
$$

But $\lambda\left(g_{i}\right)=\lambda\left(g_{i}^{\prime}\right)$, and $\gamma\left(g_{i}\right) \neq \gamma\left(g_{i}^{\prime}\right)$, so $\lambda\left(h_{i}\right)=0$ for all $\lambda \in \Pi_{p}$. Hence:

$$
\gamma\left(h_{i}\right)=(\beta * \lambda)\left(h_{i}\right)=\beta\left(g_{i}\right) \lambda\left(h_{i}\right)+\beta\left(h_{i}\right) \lambda\left(g_{i}\right)=\beta\left(h_{i}\right)
$$

The proof can now be completed:

$$
\begin{aligned}
\Sigma_{\Pi}(\mathbf{h}) & =\sum_{\beta \in \Pi^{\prime}, \lambda \in \Pi_{p}} \prod_{i \in J} \beta\left(g_{i}\right) \lambda\left(h_{i}\right) \cdot \prod_{i \notin J} \beta\left(h_{i}\right) \\
& =\sum_{\beta \in \Pi^{\prime}, \lambda \in \Pi_{p}} \beta\left(g_{J} h_{[n] \backslash J}\right) \lambda\left(h_{J}\right)=\Sigma_{\Pi^{\prime}}\left(g_{J} h_{[n] \backslash J}\right) \cdot \Sigma_{\Pi_{p}}\left(h_{J}\right)
\end{aligned}
$$

as claimed.

We compute $\Sigma_{\Pi_{p}}(\mathbf{h})$ in a later section. For now, we mention how to compute the other factor. 
Theorem 6.3. Suppose $\operatorname{char}(R) \nmid|\Pi|$, and no $h_{i}$ is pseudo-primitive with respect to (the Abelian group of weights) П. For each $i$, let $f_{i} \in k(R)$ denote $\beta\left(h_{i}\right) /\left(\beta\left(g_{i}\right)-\beta\left(g_{i}^{\prime}\right)\right)$ for some $\beta \in N_{h_{i}} \cap \Pi$. Also define $S=\left\{I \subset[n]: g_{I} g_{[n] \backslash I}^{\prime} \in G_{\Pi}(H)\right\}$. Then $\Sigma_{\Pi}(\mathbf{h})=(-1)^{n}|\Pi| \prod_{i=1}^{n} f_{i} \cdot \sum_{I \in S}(-1)^{|I|}$.

Note that if $n=1$, this is a special case of the first two parts of Theorem 3.6 above. Moreover, if some $h_{i}$ is pseudo-primitive with respect to $\Pi$, then $\Sigma_{\Pi}(\mathbf{h})=0$ from Proposition 5.5 above.

Proof. Let us fix some generators $\beta_{1}, \ldots, \beta_{k}$ of $\Pi$ (by the structure theory of finite Abelian groups), so that $\Pi=\bigoplus_{j=1}^{k} \mathbb{Z} \beta_{j}$. Then (by assumption), for each $i$ there is at least one $j$ so that $\beta_{j}\left(g_{i}\right) \neq \beta_{j}\left(g_{i}\right)^{-1}$.

Now fix $i$, and compute $\beta\left(h_{i}\right)$ for arbitrary $\beta \in \Pi$. Suppose $N_{i}^{\prime}$ indexes the set of $\beta_{j}$ 's that are in $N_{h_{i}}$; then write $\beta=\beta^{\prime}+\sum_{j \in N_{i}^{\prime}} r_{j} \beta_{j}$ for some $r_{j} \geq 0$ and $\beta^{\prime} \in \bigoplus_{j \notin N_{i}^{\prime}} \mathbb{Z} \beta_{j}$.

Note by Proposition 5.5 for $\Pi \leftrightarrow \bigoplus_{j \notin N_{i}^{\prime}} \mathbb{Z} \beta_{j}$ that $\beta^{\prime}\left(h_{i}\right)=0$. So if $\beta^{\prime \prime}=\left(\beta^{\prime}\right)^{-1} \beta \in \Pi$, then

$$
\beta\left(h_{i}\right)=\beta^{\prime}\left(g_{i}\right) \beta^{\prime \prime}\left(h_{i}\right)+\beta^{\prime}\left(h_{i}\right) \beta^{\prime \prime}\left(g_{i}\right)=\beta^{\prime}\left(g_{i}\right) \beta^{\prime \prime}\left(h_{i}\right)
$$

It remains to compute the last factor above. This is done using the following claim.

Claim. Say $\beta^{\prime \prime}=\sum_{j \in N_{i}^{\prime}} r_{j} \beta_{j}$. Then $\beta^{\prime \prime}\left(h_{i}\right)=\left(\beta^{\prime \prime}\left(g_{i}\right)-\beta^{\prime \prime}\left(g_{i}^{\prime}\right)\right) f_{i}$, where $f_{i}$ is defined in the statement of the theorem.

Proof of the theorem, modulo the claim. By the claim,

$$
\beta\left(h_{i}\right)=\beta^{\prime}\left(g_{i}\right)\left(\beta^{\prime \prime}\left(g_{i}\right)-\beta^{\prime \prime}\left(g_{i}^{\prime}\right)\right) f_{i}=\left(\beta\left(g_{i}\right)-\beta\left(g_{i}^{\prime}\right)\right) f_{i}
$$

(for all $i$ ) since $\beta^{\prime}\left(g_{i}\right)=\beta^{\prime}\left(g_{i}^{\prime}\right)$ by pseudo-primitivity. Using the notation that $\beta_{\mathbf{r}}=\sum_{j=1}^{k} r_{j} \beta_{j} \in \Pi$, one can compute $\Sigma_{\Pi}(\mathbf{h})$ to be

$$
\begin{aligned}
& =\sum_{\mathbf{r}} \prod_{i=1}^{n}\left(\beta_{\mathbf{r}}\left(g_{i}\right)-\beta_{\mathbf{r}}\left(g_{i}^{\prime}\right)\right) f_{i}=\prod_{i=1}^{n} f_{i} \cdot \sum_{\mathbf{r}} \sum_{I \subset[n]}(-1)^{n-|I|} \beta_{\mathbf{r}}\left(\prod_{i \in I} g_{i} \prod_{j \notin I} g_{j}^{\prime}\right) \\
& =(-1)^{n} \prod_{i=1}^{n} f_{i} \cdot \sum_{I \subset[n]}(-1)^{|I|} \Sigma_{\Pi}\left(g_{I} g_{[n] \backslash I}^{\prime}\right)=(-1)^{n} \prod_{i=1}^{n} f_{i} \cdot \sum_{I \subset[n]}(-1)^{|I|} \delta_{I \in S}|\Pi|
\end{aligned}
$$

by Proposition 2.8, where the last $\delta$ is 1 if $I \in S$, and 0 otherwise.

The proof is completed by showing the claim.

Proof of the claim. By Equation (3.5), and Lemma 3.8,

$$
\beta_{j}^{* r_{j}}\left(h_{i}\right)=\left(\beta_{j}\left(g_{i}\right)^{r_{j}}-\beta_{j}\left(g_{i}^{\prime}\right)^{r_{j}}\right) f_{i}, \quad \forall j \in N_{i}^{\prime}
$$

Suppose without loss of generality that we relabel the set $\left\{\beta_{j}: j \in N_{i}^{\prime}\right\}$ as $\left\{\beta_{1}, \ldots, \beta_{m}\right\}$ (i.e., relabel the generators $\beta_{j}$ of $\Pi$ so that these are before the others). Now compute the expression using the above equation:

$$
\beta^{\prime \prime}\left(h_{i}\right)=\left(\sum_{j=1}^{m} r_{j} \beta_{j}\right)\left(h_{i}\right)=\sum_{j=1}^{m} \prod_{l<j} \beta_{l}\left(g_{i}\right)^{r_{l}} \cdot\left(\beta_{j}\left(g_{i}\right)^{r_{j}}-\beta_{j}\left(g_{i}^{\prime}\right)^{r_{j}}\right) f_{i} \cdot \prod_{l>j} \beta_{l}\left(g_{i}^{\prime}\right)^{r_{l}}
$$

and this telescopes to $f_{i} \cdot \prod_{j} \beta_{j}\left(g_{i}\right)^{r_{j}}-f_{i} \cdot \prod_{j} \beta_{j}\left(g_{i}^{\prime}\right)^{r_{j}}=\left(\beta^{\prime \prime}\left(g_{i}\right)-\beta^{\prime \prime}\left(g_{i}^{\prime}\right)\right) f_{i}$, as claimed. 


\section{Products of Pseudo-Primitive Elements: Positive Characteristic}

We now mention results for pseudo-primitive elements $h_{i}$ (and not necessarily Abelian П) in prime characteristic; note that for almost all characteristics (including zero), Proposition 5.5 above says that $\Sigma_{\Pi}(\mathbf{h})=0$. Before considering the positive case, we need a small result.

Lemma 7.1. Given $f \in \mathbb{N}$ and a prime $p>0$, define $\varphi_{p}(f)=\varphi_{p}(f):=\sum_{i=0}^{p-1} i^{f}$. If $f>0$, then $\varphi(f) \not \equiv 0 \bmod p$ if and only if $(p-1) \mid f$, and in this case, $\varphi(p-1)=p-1 \equiv-1 \bmod p$.

Proof. Let $g$ be any cyclic generator of (the finite cyclic group) $(\mathbb{Z} / p \mathbb{Z})^{\times}$. Then $\sum_{i=1}^{p-1} i^{f} \equiv \sum_{j=1}^{p-1} g^{j f}$ $\bmod p$. Now if $(p-1) \mid f$, then each summand is 1 , which yields $p-1 \bmod p$. Otherwise, $g^{f}$ is not 1 , and its powers add up to 0 (by the geometric series formula).

\subsection{Preliminaries}

Recall that a pseudo-primitive element is any $h \in H_{g, g^{\prime}}$ so that $g^{-1} g^{\prime} \in G_{\Pi}(H)$. Some terminology is now needed. Note by Hall's theorems that a finite group $\Phi$ is solvable if and only if it contains Hall subgroups of all possible orders (e.g., see $[15, \S 11])$. So if $|\Phi|=p^{k} \cdot m$ with $p \nmid m$, let $\Phi_{m}$ be any Hall subgroup of order $m$.

Definition 7.2. ( $p>0$ a fixed prime.) Given a finite solvable group $\Phi$, denote by $\Phi_{p}, \Phi^{\prime}$ respectively, any $p$-Sylow subgroup and any Hall subgroup of order $|\Phi| /\left|\Phi_{p}\right|$. (From above, we mean $\Phi^{\prime}=\Phi_{m}$.)

For the rest of this section, $\operatorname{char}(R)=p>0$; also fix $n$, the number of $h_{i}$ 's. (Recall Assumption 5.1.)

Proposition 7.3. Suppose, given skew-primitive $h_{i} \in H_{g_{i}, g_{i}^{\prime}}$ for $1 \leq i \leq n$, that $\Phi \subset \Gamma / \Gamma^{\prime}$ is a finite solvable subquotient of $\Gamma$ (as in a previous section) with respect to which every $h_{i}$ is pseudo-primitive. Then $\Sigma_{\Phi}(\mathbf{h})=\Sigma_{\Phi^{\prime}}(\mathbf{g}) \cdot \Sigma_{\Phi_{p}}(\mathbf{h})$.

Proof. The first claim is that the "set-product" $\Phi^{\prime} \Phi_{p}:=\left\{\beta * \lambda: \beta \in \Phi^{\prime}, \lambda \in \Phi_{p}\right\}$ equals the entire group $\Phi$. Next, if $\beta \in \Phi^{\prime}$, then $\beta(h)=0$ for any pseudo-primitive $h \in H_{g, g^{\prime}}$, because if $\left|\Phi^{\prime}\right|=m \not \equiv 0$ $\bmod p$, then $\beta^{* m}=\varepsilon \in \Gamma^{\prime} \subset \Gamma_{h}$, whence $0=\varepsilon(h)=\beta^{* m}(h)=m \beta(g)^{m-1} \beta(h)$. Therefore,

$$
\begin{aligned}
\Sigma_{\Phi}(\mathbf{h}) & =\sum_{\beta \in \Phi^{\prime}, \lambda \in \Phi_{p}} \prod_{i=1}^{n}(\beta * \lambda)\left(h_{i}\right)=\sum_{\beta \in \Phi^{\prime}, \lambda \in \Phi_{p}} \prod_{i=1}^{n} \beta\left(g_{i}\right) \lambda\left(h_{i}\right) \\
& =\sum_{\beta \in \Phi^{\prime}} \prod_{i=1}^{n} \beta\left(g_{i}\right) \cdot \sum_{\lambda \in \Phi_{p}} \prod_{i=1}^{n} \lambda\left(h_{i}\right)=\Sigma_{\Phi^{\prime}}(\mathbf{g}) \Sigma_{\Phi_{p}}(\mathbf{h})
\end{aligned}
$$

as claimed.

Remark 7.4. The above proposition thus holds for any group $\Phi$, such that some $p$-Sylow subgroup $\Phi_{p}$ has a complete set of coset representatives, none of whom has order divisible by $p$. Obvious examples are Abelian groups or groups of order $p^{a} q^{b}$ for primes $p \neq q$ (but these are solvable by Burnside's Theorem).

Also note that the above sum is independent of the choices of $\Phi_{p}, \Phi^{\prime}$.

The next result is crucial in computing $\Sigma_{\Pi}(\mathbf{h})$, and uses subquotients of $\Pi$. 
Theorem 7.5. Given a finite subgroup of weights $\Pi \subset \Gamma=\Gamma_{H}$, suppose $h_{i} \in H_{g_{i}, g_{i}^{\prime}}$ is pseudo-primitive with respect to $\Pi$ for all $1 \leq i \leq n$. Define $\Phi=\Pi_{a b}:=\Pi /[\Pi, \Pi]$. Then,

$$
\Sigma_{\Pi}(\mathbf{h})=|[\Pi, \Pi]| \cdot \Sigma_{\Phi^{\prime}}(\mathbf{g}) \cdot \Sigma_{\Phi_{p}}(\mathbf{h})
$$

For instance, if every $h_{i}$ was almost primitive, then $\Sigma_{\Phi^{\prime}}(\mathbf{g})=\left[\Phi: \Phi_{p}\right]$.

Proof. At the outset, note that $\Sigma_{\Phi^{\prime}}(\mathbf{g})$ and $\Sigma_{\Phi_{p}}(\mathbf{h})$ make sense because of Corollary 4.6 and Proposition 4.2 above. Now, the proof is in two steps; each step uses a previously unused result above.

Step 1. We claim that $\Sigma_{\Pi}(\mathbf{h})=|[\Pi, \Pi]| \Sigma_{\Phi}(\mathbf{h})$. This follows immediately from Lemma 4.4, where $h, \Gamma^{\prime \prime}, \Pi^{\circ}$ are replaced by $\mathbf{h},[\Pi, \Pi], \Pi$ respectively.

The only thing to check is that the above replacements are indeed valid. Since $[\Pi, \Pi]$ is normal in $\Pi$, it suffices to check that $[\Pi, \Pi] \subset \Gamma_{\mathbf{h}}$. But this follows from Corollary 4.6 and Proposition 4.2 above.

Step 2. The proof is now complete by invoking Proposition 7.3 above.

We conclude the preliminaries with one last result-for skew-primitive elements in general.

Proposition 7.7. If $\Pi_{p} \nsucceq(\mathbb{Z} / p \mathbb{Z})^{k}$ for any $k>0$, then $\Sigma_{\Pi_{p}}(\mathbf{h})=0$.

Proof. By Theorem 5.6, the $h_{i}$ 's are almost primitive with respect to $\Pi_{p}$. Now invoke Equation (7.6) above, replacing $\Pi$ by $\Pi_{p}$. Now, if $\Pi_{p}$ is not Abelian, then $\left|\left[\Pi_{p}, \Pi_{p}\right]\right|>1$, hence is a power of $p$, whence the right-hand side vanishes. Next, if $\Pi_{p}$ is Abelian, but contains an element of order $p^{2}$, then $\Sigma_{\Pi_{p}}(\mathbf{h})=0$ by Theorem 5.6 again. Therefore $\Pi_{p} \cong(\mathbb{Z} / p \mathbb{Z})^{k}$ for some $k$. If $k=0$, then $\Pi_{p}=\{\varepsilon\}$, and $\varepsilon(\mathbf{h})=0$.

\subsection{The Main Results-Pseudo-Primitive Elements}

The following result now computes $\Sigma_{\Pi}(\mathbf{h})$ (for pseudo-primitive $h_{i}$ 's) in most cases in prime characteristic that are "non-Abelian". For the "Abelian" case, we appeal to Theorem 7.10 below and mention at the outset that it is only for almost primitive (and not merely pseudo-primitive) elements, that we obtain a much clearer picture-as its last part shows.

Theorem 7.8. Suppose $\operatorname{char}(R)=p \in \mathbb{N}, \Pi_{p}$ is any (fixed) p-Sylow subgroup of $\Pi$, and every $h_{i}$ is pseudo-primitive (with respect to $\Pi$ ).

1. $\Sigma_{\Pi}(\mathbf{h})=0$ if $\Pi_{p}$

(a) is trivial,

(b) contains an element of order $p^{2}$, or

(c) intersects $[\Pi, \Pi]$ nontrivially.

This last part includes the cases when $\Pi_{p}$

(d) is not Abelian,

(e) does not map isomorphically onto (some) $\Phi_{p}$, via (the restriction of) the quotient map $\pi$ : $\Pi \rightarrow \Phi=\Pi /[\Pi, \Pi]$, or

(f) has size strictly greater than $\Phi_{p}$.

2. Otherwise $\Pi_{p} \cong \Phi_{p} \cong(\mathbb{Z} / p \mathbb{Z})^{k}$ for some $k>0$, and then $\Sigma_{\Pi}(\mathbf{h})=|[\Pi, \Pi]| \cdot \Sigma_{\Phi^{\prime}}(\mathbf{g}) \cdot \Sigma_{\Pi_{p}}(\mathbf{h})$. 
Remark 7.9. Any finite Abelian group of exponent $p$ is of the form $(\mathbb{Z} / p \mathbb{Z})^{k}$, hence one part of the second statement is clear. Moreover, every subquotient of such a group is of the same form. Finally (especially when all of the $h_{i}$ 's are almost primitive with respect to $\Pi$ ), the cases that remain reduce to computing $\Sigma_{\Pi_{p}}(\mathbf{h})$, and when $\Pi_{p} \cong(\mathbb{Z} / p \mathbb{Z})^{k}$; this is addressed below.

Proof. The second part follows from the first part, the remarks above, and Equation (7.6). We now show the first part.

(a) If $\Pi_{p}$ is trivial, then $p \nmid|\Pi|$, and we are done by Proposition 5.5.

(b) This has been done in Theorem 5.6 above.

(c) Now suppose that $[\Pi, \Pi] \cap \Pi_{p} \neq \emptyset$. Then $[\Pi, \Pi]$ contains an element of order $p$, whence $p$ divides $|[\Pi, \Pi]|$. Now use Equation (7.6).

It remains to show how this last includes the remaining cases.

(d) First, if $\Pi_{p}$ is non-Abelian, then $\left[\Pi_{p}, \Pi_{p}\right]$ is a nontrivial subgroup of the $p$-group $\Pi_{p}$. In particular, $\Pi_{p}$ intersects $[\Pi, \Pi]$.

(e) Next, note that $\pi\left(\Pi_{p}\right)$ is a $p$-group in $\Phi$, and $\left|\Pi_{p}\right| \geq\left|\Phi_{p}\right|$ (since $|\Phi|$ divides $|\Pi|$ ). Hence $\Pi_{p}$ does not map isomorphically onto (some) $\Phi_{p}$ if and only if $\pi$ is not one-to-one on $\Pi_{p}$. But then $[\Pi, \Pi]$ intersects $\Pi_{p}$.

(f) Finally, if $\left|\Pi_{p}\right|>\left|\Phi_{p}\right|$, then $\Pi_{p}$ cannot map isomorphically onto $\Phi_{p}$, so we are done by the preceding paragraph.

We conclude by analyzing $\Sigma_{\Pi_{p}}(\mathbf{h})$. Note that the results below that pertain only to $\Sigma_{\Pi_{p}}(\mathbf{h})$ are applicable in general to all skew-primitive $h_{i}$ 's, by Theorem 5.6 above.

Theorem 7.10. Suppose $\operatorname{char}(R)=p \in \mathbb{N}, \Pi_{p}$ is any (fixed) p-Sylow subgroup of $\Pi$, and every $h_{i} \in$ $H_{g_{i}, g_{i}^{\prime}}$ is pseudo-primitive (with respect to $\Pi$ ) for all $1 \leq i \leq n$. Suppose moreover that $\Pi_{p} \cong(\mathbb{Z} / p \mathbb{Z})^{k}$.

1. $\gamma\left(g_{i}\right)=1$ for all $i$ and $\gamma \in \Pi_{p}$. In particular, $g_{i}, \mathbf{g} \in G_{\Pi_{p}}(H)$.

2. If $k>n$, then $\Sigma_{\Pi}(\mathbf{h})=\Sigma_{\Pi_{p}}(\mathbf{h})=0$.

3. If $k=n$, then $\Sigma_{\Pi_{p}}(\mathbf{h})=\left(\begin{array}{l}p \\ 2\end{array}\right)^{k} \cdot \operatorname{perm}(A)$, where $A$ is the matrix given by $a_{i j}=\gamma_{j}\left(h_{i}\right)$, the $\gamma_{j}$ 's form $a \mathbb{Z} / p \mathbb{Z}$-basis of $\Pi_{p}$, and perm is the matrix permanent:

$$
\operatorname{perm}\left(A_{n \times n}\right)=\sum_{\sigma \in S_{n}} \prod_{i=1}^{n} a_{i, \sigma(i)}
$$

In particular, $\Sigma_{\Pi_{p}}(\mathbf{h})=0$ unless $p=2$, in which case $\Sigma_{\Pi}(\mathbf{h})=\Sigma_{\Pi_{p}}(\mathbf{h})=\operatorname{det} A$.

4. If $\Sigma_{\Pi_{p}}(\mathbf{h}) \neq 0$, then $(p-1) \mid n$ and $0<k \leq n /(p-1)$, and then $\Sigma_{\Pi_{p}}(\mathbf{h})$ can take any value $r \in R$. (If $k=n$ and $p=2$, then $r \neq 0$.)

Remark 7.11. This result is independent of the chosen $p$-Sylow subgroup $\Pi_{p}$, as well as the choices of generators $\gamma_{j}$. It generalizes Proposition 3.9 above, in the special case $p=2$. 
The rest of this section is devoted to proving the above result. First, suppose there exists a subgroup $\Pi_{p}^{\prime} \cong(\mathbb{Z} / p \mathbb{Z})^{k}$ of $\Pi$ (so $\Pi_{p}^{\prime} \subset \Pi_{p}$ in general). Choose a set of coset representatives $\mathcal{B}$ for $\Pi_{p}^{\prime}$ in $\Pi$, and write

$$
\Sigma_{\Pi}(\mathbf{h})=\sum_{\beta \in \mathcal{B}, \gamma \in \Pi_{p}^{\prime}}(\gamma * \beta)(\mathbf{h})=\sum_{\beta \in \mathcal{B}, \gamma \in \Pi_{p}^{\prime}} \prod_{i=1}^{n}(\gamma * \beta)\left(h_{i}\right)
$$

Recall that every element of $\Pi_{p}^{\prime}$ is $\gamma_{a, I}:=\sum_{j \in I} a_{j} \gamma_{j}$ (with $\gamma_{i}$ as above), for some subset $I$ of $[k]:=\{1,2, \ldots, k\}$, and some $|I|$-tuple $a=\left(a_{j}\right)_{j \in I}$ of elements of $(\mathbb{Z} / p \mathbb{Z})^{\times}$. Recall, moreover, that we had previously defined $g_{I}, g_{I}^{\prime}, h_{I}$ for $I \subset[n]$.

We also need the following lemma that is proved by computations using Proposition 4.5.

Lemma 7.12. If $h \in H_{g, g^{\prime}}$ is pseudo-primitive with respect to $\Pi$, and given $\beta \in \mathcal{B}, \gamma_{a, I} \in \Pi_{p}$ as above, one has

$$
\left(\beta * \gamma_{a, I}\right)(h)=\prod_{j=1}^{k} \gamma_{j}(g)^{a_{j}} \cdot\left[\beta(h)+\beta(g) \sum_{j=1}^{k} a_{j} \gamma_{j}\left(g^{-1} h\right)\right]
$$

The key observation now is that the only "monomials" that occur in the product $\prod_{i=1}^{n}\left(\beta * \gamma_{a, I}\right)\left(h_{i}\right)$ are of the form $\beta\left(\mathbf{g} g_{I_{0}}^{-1} h_{I_{0}}\right) \cdot \prod_{j \in I} \gamma_{j}\left(h_{I_{j}}\right)$, where $\coprod_{j} I_{j} \coprod_{0}=[n]$, and $I_{j} \subset I$ for all $j$. The coefficient of such a monomial in this particular summand is $\prod_{j \in I} a_{j}^{\left|I_{j}\right|} \gamma_{j}(\mathrm{~g})^{a_{j}}$ by the lemma above. Moreover, every such monomial occurs at most once inside each $\left(\beta * \gamma_{a, I}\right)(\mathbf{h})$.

The crucial fact that proves Theorem 7.10 above is the following

Key claim. The coefficient of $\beta\left(\mathbf{g} g_{I_{0}}^{-1} h_{I_{0}}\right) \prod_{j \in I} \gamma_{j}\left(h_{I_{j}}\right)$ in $\sum_{\gamma \in \Pi_{p}^{\prime}}(\beta * \gamma)(\mathbf{h})$ equals $p^{k-|I|} \prod_{j \in I} \varphi\left(\left|I_{j}\right|\right)$ (where $\varphi(0):=0)$.

Proof of the key claim. (Note that $\varphi(f)$ was defined in Lemma 7.1.) A monomial of the desired form occurs in precisely those $\left(a^{\prime}, I^{\prime}\right)$-summands, so that $I^{\prime} \supset I$. Moreover, all such summands can be split up into a disjoint union over all $a \in\left((\mathbb{Z} / p \mathbb{Z})^{\times}\right)^{|I|}$, with each disjoint piece containing all $\left(a^{\prime}, I^{\prime}\right)$ so that $I^{\prime} \supset I$ and the $I$-component of $a^{\prime}$ is $a$.

Such a piece contains exactly $p^{k-|I|}$ elements (and hence exactly that number of copies of the monomial with this selfsame coefficient). Each of these "extra" $[k] \backslash I$ factors contributes a $\beta\left(h_{i}\right)$, which gives $\beta\left(h_{I_{0}}\right)$.

Moreover, there is one contribution for each $a \in\left((\mathbb{Z} / p \mathbb{Z})^{\times}\right)^{|I|}$, and it is $\prod_{j \in I} a_{j}^{\left|I_{j}\right|} \gamma_{j}(\mathbf{g})^{a_{j}}$. $\beta\left(\mathbf{g} g_{I_{0}}^{-1}\right)$, since the argument for the $\beta$-factor here is precisely $\prod_{j=1}^{k} g_{I_{j}}$. Moreover, $\gamma_{j}(\mathbf{g})=1$ by Theorem 5.6 above.

Summing over all possible tuples $a \in\left((\mathbb{Z} / p \mathbb{Z})^{\times}\right)^{|I|}$, the coefficient (apart from the $\beta$-part) is

$$
p^{k-|I|} \sum_{a} \prod_{j \in I} a_{j}^{\left|I_{j}\right|}=p^{k-|I|} \prod_{j \in I} \sum_{a_{j}=1}^{p-1} a_{j}^{\left|I_{j}\right|}
$$

and this equals $p^{k-|I|} \prod_{j \in I} \varphi\left(\left|I_{j}\right|\right)$ as desired, because the only problem may occur when some $\left|I_{j}\right|=0$. But then $|I|<k$, so

$$
p^{k-|I|} \sum_{a_{j}=1}^{p-1} a_{j}^{0}=0 \sum_{a_{j}=1}^{p-1} a_{j}^{0}=0 \sum_{a_{j}=1}^{p} a_{j}^{0}=p^{k-|I|} \varphi(0)
$$




\section{Proof of Theorem 7.10.}

1. This is from Theorem 5.6 above.

Now set $\Pi_{p}=\Pi_{p}^{\prime}$. We first note from the key claim that if $I_{0}$ is nonempty, or any $I_{j}$ is empty, then the coefficient of that particular monomial vanishes- because $\operatorname{char}(R)=p$ and $\varphi(0)=0$.

2. Suppose $k>n$. Then at least one $I_{j}$ must be empty in every monomial above, by the Pigeonhole Principle, and we are done.

3. If $k=n$, then the only monomials that have a nonzero contribution to the sum $\Sigma_{\Pi_{p}^{\prime}}(\mathbf{h})$ must correspond to empty $I_{0}$ and singleton $I_{j}$ 's (since $\coprod_{j=1}^{k} I_{j}=[n]=[k]$ ). In other words, $\sigma \in S_{n}$ : $j \mapsto i_{j} \forall j$. Moreover, the coefficient of such a monomial is $p^{0} \prod_{j=1}^{n} \varphi(1)$, and these monomials all add up to give the matrix permanent, as claimed. The rest of the statements are now easy to see.

4. In this part, we are only concerned with $\Sigma_{\Pi_{p}}(\mathbf{h})$, so that $\beta$ does not contribute here either (so $I_{0}=\emptyset$ and $[n]=\coprod_{j \in I} I_{j}$ ).

From the key claim and Lemma 7.1 above, observe that if some monomial has a nonzero contribution, then $(p-1)$ divides $\left|I_{j}\right|$ for all $j$, and $I=[k]$. In particular, $(p-1)$ divides $\sum_{j \in I}\left|I_{j}\right|=n$, and

$$
n=\sum_{j \in I}\left|I_{j}\right|=\sum_{j=1}^{k}\left|I_{j}\right| \geq \sum_{j=1}^{k}(p-1)=k(p-1)
$$

whence $k \leq n /(p-1)$. Moreover, $\Sigma_{\Pi_{p}}(\mathbf{h})=\varepsilon(\mathbf{h})=0$ if $k=0$.

It remains to present, for each $0<k \leq n /(p-1)$ and (nonzero) $r \in R$, an example of $\left(H, \Pi=\Pi_{p}\right)$, so that $\Sigma_{\Pi}(\mathbf{h})=\Sigma_{\Pi_{p}}(\mathbf{h})=r$. This example is analyzed in the next section.

\section{Example: Lie Algebras}

Suppose $H=U(\mathfrak{g})$ for some Lie algebra $\mathfrak{g}$ (say over $\mathbb{C}$ ). Then any weight $\mu \in \Gamma_{H}$ kills $[\mathfrak{g}, \mathfrak{g}]$, hence belongs to $(\mathfrak{g} /[\mathfrak{g}, \mathfrak{g}])^{*}$. Let us denote $\mathfrak{g}_{a b}:=\mathfrak{g} /[\mathfrak{g}, \mathfrak{g}]$. Conversely, any element of the set above, is a weight of $H$, using multiplicativity and evaluating it at the projection down to the quotient $\mathfrak{g}_{a b}$. Thus, $\Gamma_{H}$ is the dual space (under addition) of the abelianization $\mathfrak{g}_{a b}$ of $\mathfrak{g}$. Hence we now examine what happens in the case of an $(R$-free) Abelian Lie algebra $\mathfrak{h}$.

In this case, we have the free $R$-module $\mathfrak{h}=\oplus_{i} R h_{i}$ with the trivial Lie bracket, and $H=U(\mathfrak{h})=\operatorname{Sym}(\mathfrak{h})$. Thus, $H$ inherits the usual Hopf algebra structure now (i.e., $\Delta\left(h_{i}\right)=1 \otimes h_{i}+$ $\left.h_{i} \otimes 1, S\left(h_{i}\right)=-h_{i}, \varepsilon\left(h_{i}\right)=0 \forall i\right)$.

First, $\left(\Gamma_{H}, *\right)=\left(\mathfrak{h}^{*},+\right)$. By Proposition 5.5, if $\operatorname{char}(R) \nmid|\Pi|$, then $\Sigma_{\Pi}(\mathbf{h})=0$ for all products $\mathbf{h}$ of primitive elements in $H$ (and hence for all $\mathbf{h}$ in the augmentation ideal $\mathfrak{h} U(\mathfrak{h})$ of $H$ ). Thus, the only case left to consider is when $\operatorname{char}(R)=p>0$. But then $\left(\mathfrak{h}^{*},+\right)$ is a $\mathbb{Z} / p \mathbb{Z}$-vector space, so every finite subgroup $\Pi$ is of the form $\Pi=\Pi_{p} \cong(\mathbb{Z} / p \mathbb{Z})^{k}$ for some $k$. Moreover, Theorem 3.6 and (the last part of) Theorem 7.10 provide more results in this case.

We therefore conclude the example (and the proof of the theorem above) by analyzing the computation of $\Sigma_{\Pi}(\mathbf{h})$ for $\mathbf{h}=h_{1} \ldots h_{n}$. For any (nonzero) $r \in R$, we produce such a finite subgroup $\Pi=\Pi_{p} \cong(\mathbb{Z} / p \mathbb{Z})^{k}$, so that $0<k \leq n /(p-1)$ and $\Sigma_{\Pi}(\mathbf{h})=r$. 
Construction: Given $k$, partition $[n]$ into $k$ disjoint nonempty subsets $[n]=\coprod_{j=1}^{k} I_{j}$, reordered so that $I_{1}=\{1, \ldots, n-(k-1)(p-1)\}$, and so that $\left|I_{j}\right|=p-1$ for all $j>1$. For each $1 \leq j \leq n$, define $\gamma_{j} \in \mathfrak{h}^{*}=\Gamma_{H}$ as follows: $\gamma_{1}\left(h_{1}\right)=r, \gamma_{j}\left(h_{i}\right)=1$ if $i \neq 1 \in I_{j}$, and $\gamma_{j}\left(h_{i}\right)=0$ otherwise. (One verifies that the $\gamma_{i}$ 's thus defined are indeed linearly independent over $k(R)$, hence over $\mathbb{Z} / p \mathbb{Z}$ as well, but for this, one needs that $r \neq 0$ if $n=k, p=2$.) Thus for any $K \subset[k], \gamma_{K}\left(h_{i}\right):=\sum_{j \in K} \gamma_{j}\left(h_{i}\right)$ vanishes unless $i \in \cup_{j \in K} I_{j}$.

Now evaluate $\Sigma_{\Pi}(\mathbf{h})=\sum_{K \subset[k], a} \prod_{i=1}^{n} \gamma_{a, K}\left(h_{i}\right)$, where $\Pi:=\sum_{i=1}^{n} \mathbb{Z} \gamma_{i}=\bigoplus_{i=1}^{n}(\mathbb{Z} / p \mathbb{Z}) \gamma_{i}$. By the key claim in the previous section, the only monomials $\prod_{j \in I} \gamma_{j}\left(h_{I_{j}^{\prime}}\right)$ that do not vanish are for $|I|=k$, and with $(p-1)$ divides $\left|I_{j}^{\prime}\right|$ for all $j$. Moreover, $\gamma_{j}\left(h_{i}\right)$ is zero except when $i \in I_{j}$, so there is only one type of monomial remaining: $\prod_{j \in I} \gamma_{j}\left(h_{I_{j}}\right)$. (Note that this satisfies the earlier condition: $(p-1)$ divides $\left|I_{j}\right|$ for all $j$.)

Moreover, by the key claim in the preceding section, the coefficient of this monomial, which itself equals $r \cdot \prod_{i=2}^{n} 1=r$, is $\prod_{j=1}^{k} \varphi\left(\left|I_{j}\right|\right)$, and by Fermat's Little Theorem, $\varphi\left(\left|I_{j}\right|\right)=p-1=-1 \forall j$ (in characteristic $p$ ). We conclude that $\Sigma_{\Pi}(\mathbf{h})=\Sigma_{\Pi_{p}}(\mathbf{h})=(-1)^{k} r$, whence we are done (start with $r^{\prime}=(-1)^{k} r$ to get $\left.r\right)$.

\section{Example: Degenerate Affine Hecke Algebras of Reductive Type with Trivial Parameter}

In this section, we apply the general theory above, to a special case, wherein a finite group acts on a vector space (or free $R$-module in our case), with the group and the module corresponding to the Weyl group and the Cartan subalgebra (actually, its dual space) respectively, of a reductive Lie algebra. We use the $\mathbb{Z}$-basis of simple roots (and any $\mathbb{Z}$-basis for the center), to try and compute the value of $\Sigma_{\Pi}(\mathbf{h})$.

\subsection{Hopf Algebras Acting on Vector Spaces}

We will consider special cases of the following class of Hopf algebras. Suppose that a cocommutative $R$-Hopf algebra $H$ acts on a free $R$-module $V$; denote the action by $h \cdot v$ for $h \in H, v \in V$. Then $H$ also acts on $V^{*}$ by: $\langle h \cdot \lambda, v\rangle:=\langle\lambda, S(h) \cdot v\rangle$.

Now consider the $R$-algebra $A$ generated by the sets $H$ and $V$, with obvious relations in $H$, and the extra relations $v v^{\prime}=v^{\prime} v, \sum h_{(1)} v S\left(h_{(2)}\right)=: \operatorname{ad} h(v)=h \cdot v$ for all $h \in H$ and $v, v^{\prime} \in V$. Note that the relation ad $h(v)=h \cdot v$ can be rephrased, as the following lemma shows.

Lemma 9.1. Suppose some R-Hopf algebra $H$ acts on a free $R$-module $V$, and an $R$-algebra $B$ contains $H, V$. Then the following relations are equivalent (in $B$ ) for all $v \in V$ :

1. $\sum h_{(1)} v S\left(h_{(2)}\right)=h \cdot v$ for all $h \in H$.

2. $h v=\sum\left(h_{(1)} \cdot v\right) h_{(2)}$ for all $h \in H$.

If $H$ is cocommutative, then both of these are also equivalent to:

3. $v h=\sum h_{(1)}\left(S\left(h_{(2)}\right) \cdot v\right)$ for all $h \in H$.

Moreover, if this holds, then any unital subalgebra $M$ of $B$ that is also an $H$-submodule (via ad) is an $H$-(Hopf-)module algebra under the action

$$
h \cdot m:=\operatorname{ad} h(m)=\sum h_{(1)} m S\left(h_{(2)}\right) \forall h \in H, m \in M
$$


(The proof is straightforward.) For instance, one can take $M=B$ or $H-$ or in the above example of $A$, consider $M=\operatorname{Sym}_{R} V$.

It is straightforward (but perhaps tedious) to check that $A$ is a Hopf algebra with the usual operations: on $H$, they restrict to the Hopf algebra structure of $H$, and $V$ consists of primitive elements.

By the above lemma, if $H$ is $R$-free, then the ring $A$ is an $R$-free $R$-Hopf algebra, with $R$-basis given by $\{h \cdot m\}$, where $h \in H$ and $m$ run respectively over some $R$-basis of $H$, and all (monomial) words (including the empty word) with alphabet given by an $R$-basis of $V$. It has the subalgebras $H$ and $\operatorname{Sym}_{R}(V)$, and is called the smash product $H \ltimes \operatorname{Sym}_{R} V$ of $H$ and $\operatorname{Sym}_{R} V$.

We now determine the weights of $A$. Denote by $\Gamma_{H}$ the group of weights of $H$ (under convolution). One can now use Proposition 2.13 to prove:

Proposition 9.2. The weights $\Gamma_{A}$ of $A$ form a group, which is the Cartesian product $\Gamma_{H} \times V_{\varepsilon}^{*}$, with convolution given by

$$
\left(\nu_{1}, \lambda_{1}\right) *\left(\nu_{2}, \lambda_{2}\right)=\left(\nu_{1} \nu_{2}, \lambda_{1}+\lambda_{2}\right)=\left(\nu_{1} *_{H} \nu_{2}, \lambda_{1} *_{V} \lambda_{2}\right)
$$

for $\nu_{i} \in \Gamma_{H}, \lambda_{i} \in V_{\varepsilon}^{*}$. (Here, $V_{\varepsilon}^{*}$ is the $\varepsilon$-weight space of the $H$-module $V^{*}$.)

\subsection{Degenerate Affine Hecke Algebras}

Since we work over any commutative unital integral domain $R$, we can generate examples over all $R$ if there exists a lattice in $V$ that is fixed by $H$, and one considers its $R$-span. Now specialize to the case when $H=R W$ is the group ring of a Weyl group acting on a Cartan subalgebra of the corresponding semisimple Lie algebra. Then one uses the root lattice $Q$ inside $V=\mathfrak{h}^{*}$.

We work in slightly greater generality. Given a finite-dimensional reductive complex Lie algebra $\mathfrak{g}$, let $W$ be its Weyl group and $\mathfrak{h}$ a fixed chosen Cartan subalgebra. Thus $\mathfrak{h}=\oplus_{i \geq 0} \mathfrak{h}_{i}$, where for $i>0, \mathfrak{h}_{i}$ corresponds to a simple component (ideal) of $\mathfrak{g}$, with corresponding base of simple roots $\Delta_{i}$ and Weyl group $W_{i}$, say; and $\mathfrak{h}_{0}$ is the central ideal in $\mathfrak{g}$.

Define $Q_{i}=\oplus_{\alpha \in \Delta_{i}} \mathbb{Z} \alpha$, the root lattice inside $\mathfrak{h}_{i}^{*}$, and choose and fix some $\mathbb{Z}$-lattice $Q_{0}$ inside $\mathfrak{h}_{0}^{*}$. Now replace $\mathfrak{h}_{i}^{*}$ by $V_{i}=\mathfrak{h}_{i}^{*}:=R \otimes_{\mathbb{Z}} Q_{i}$, and $\mathfrak{h}_{i}$ by the $R$-dual of $\mathfrak{h}_{i}^{*}$, for all $i \geq 0$. Thus, for the entire Lie algebra, $\Delta=\coprod_{i>0} \Delta_{i}$ and $W=\times_{i>0} W_{i}$.

Now define $V=\oplus_{i \geq 0} V_{i}$, whence the previous subsection applies and one can form the algebra $A=R W \ltimes \operatorname{Sym}_{R} V$. This is the degenerate affine Hecke algebra with trivial parameter (the parameter is trivial since $w v-w(v) w$ is always zero), of reductive type. This is a special case of [16, Definition 1.1], where one sets $\eta=0$.

Before we address the general case, note that there are two types of $\mathfrak{h}_{i}$ 's in here: ones corresponding to simple Lie algebras, which we address first, and the "central part", which is fixed by $W$ (hence so is $\mathfrak{h}_{0}^{*}$ ).

\subsection{The Simple Case}

The first case to consider is: $V=\mathfrak{h}^{*}=R \otimes_{\mathbb{Z}} Q$, for a simple Lie algebra. Thus $\Delta$ is irreducible, and given $A=R W \ltimes \operatorname{Sym}_{R}\left(\mathfrak{h}^{*}\right), \Gamma_{A}=\Gamma_{W} \times \mathfrak{h}^{W}$ (because the condition in Proposition 9.2 above translates to: $w(\gamma)=\varepsilon(w) \gamma=\gamma$ for all $\left.w \in W, \gamma \in \Gamma_{A}\right)$. Here, $\Gamma_{W}=\Gamma_{R W}$. 
We now state our main result, using the convention that all roots in the simply laced cases (types $A, D, E)$ are short. The result helps compute $\Sigma_{\Pi}$ at any element of the $R$-basis $\{g \cdot m\}$ mentioned in an earlier subsection.

Theorem 9.3. Suppose $\mathfrak{g}$ is a complex simple Lie algebra with simple roots $\Delta$, Weyl group $W$, $V=\mathfrak{h}^{*}=R \otimes_{\mathbb{Z}} \mathbb{Z} \Delta$, and $A=R W \ltimes \operatorname{Sym}_{R}\left(\mathfrak{h}^{*}\right)$. As above, let $\Pi \subset \Gamma_{A}$ be a finite subgroup of weights. Let $h_{1}, \ldots, h_{n} \in \mathfrak{h}^{*}$.

1. If $\operatorname{char}(R) \neq 2$, or $W$ is of type $G_{2}$, or $W$ has more than one short simple root, then every weight acts as $\varepsilon=0$ on $\mathfrak{h}^{*}$. In particular, $\Sigma_{\Pi}=0$ on $\operatorname{Sym}_{R}\left(\mathfrak{h}^{*}\right)$.

2. If $\operatorname{char}(R)=2$, then every weight acts as $\varepsilon$ on $W$. Now suppose also that $W$ is not of type $G_{2}$, and has only one short simple root $\alpha_{s}$, say.

If $\Pi$ has an element of order 4 , or $h_{i}$ has no " $\alpha_{s}$-contribution" (i.e., $h_{i} \in \oplus_{\alpha_{s} \neq \alpha \in \Delta} R \cdot \alpha$ ) for some $i$, then $\Sigma_{\Pi}(\mathbf{h})=0$.

3. If this does not happen, i.e., $\Pi=(\mathbb{Z} / 2 \mathbb{Z})^{k}$ for some $k$, and the hypotheses of the previous part hold, then

$$
\Sigma_{\Pi}\left(\alpha_{s}^{n}\right)=\sum_{\substack{l_{i}>0 \forall i \\
l_{1}+\cdots+l_{k}=n}}\left(\begin{array}{c}
n \\
l_{1}, \ldots, l_{k}
\end{array}\right) \prod_{i=1}^{k} \gamma_{i}\left(\alpha_{s}\right)^{l_{i}}
$$

where the $\gamma_{i}$ 's are any set of generators for $\Pi$. In particular, this vanishes if $k>r$, where $\sum_{j=1}^{r} 2^{s_{j}}$ is the binary expansion of $n$.

\section{Remark 9.4.}

1. Warning. One should not confuse the $h_{i}$ 's here with elements of $\mathfrak{h}$; indeed, $h_{i} \in A$, so they really are in $\mathfrak{h}^{*}$.

2. The coefficient above is just the multinomial coefficient $n ! /\left(\prod_{i} l_{i} !\right)$, which we also denote by $\left(\begin{array}{c}n \\ l_{1}, \ldots, l_{k-1}\end{array}\right)$, just as $\left(\begin{array}{c}n \\ k, n-k\end{array}\right)=\left(\begin{array}{l}n \\ k\end{array}\right)$. The last line in the theorem follows because this coefficient is odd if and only if ( $r, s_{j}$ as above) we can partition $\left\{2^{s_{j}}: j\right\}$ into $k$ nonempty subsets, and the $l_{i}$ 's are precisely the sums of the elements in the subsets. (This fails, for instance, if some two $l_{i}$ 's are equal, or $k>r$.) In turn, this fact follows (inductively) from the following easy-to-prove

Lemma 9.5. Suppose $p>0$ is prime, $p^{s} \leq n<p^{s+1}$ for some $s \geq 0$, and $l_{k} \geq l_{i} \forall i$. If $l_{k}<p^{s}$ then p divides $\left(\begin{array}{c}n \\ l_{1}, \ldots, l_{k-1}\end{array}\right)$. Otherwise $p$ divides neither or both of $\left(\begin{array}{c}n \\ l_{1}, \ldots, l_{k-1}\end{array}\right)$ and $\left(\begin{array}{c}n-p^{s} \\ l_{1}, \ldots, l_{k-1}\end{array}\right)$.

The rest of the subsection is devoted to the proof of the theorem. We once again mention a result crucial to the proof, then use it to prove the theorem, and conclude by proving the key claim.

Key claim. ( $\operatorname{char}(R)$ arbitrary.) If $W$ contains a Dynkin subgraph $\Omega$ of type $A_{2}$ or $G_{2}$, then both the simple roots in $\Omega$ are killed by all $\lambda \in \mathfrak{h}^{W}$. If $\Omega$ is of type $B_{2}$, then the long root in $\Omega$ is killed by all $\lambda$.

Proof modulo the key claim. We now show the theorem.

1. First suppose that $\operatorname{char}(R) \neq 2$. If $\lambda \in \mathfrak{h}^{W}$, then $\lambda(\alpha)=\lambda\left(s_{\alpha}(\alpha)\right)=-\lambda(\alpha)$, whence $\lambda(\alpha)=0$ for all $\alpha \in \Delta$, and $\mathfrak{h}^{W}=0$. 
For the other claims, use the classification of simple Lie algebras in terms of Dynkin diagrams, as mentioned in [7, Chapter 3]. To show that a weight $\lambda$ kills all of $\mathfrak{h}^{*}$, it suffices to show that $\lambda(\alpha)=0 \forall \alpha \in \Delta$, i.e., that it kills each simple root or node of the corresponding Dynkin diagram. If the Dynkin diagram of a Lie algebra has (a sub-diagram of) type $A_{2}$ or $G_{2}$, then both nodes of that diagram (or both $\alpha_{i}$ 's) are killed by all weights $\lambda \in \Gamma$, by the key claim above. This automatically eliminates all diagrams of type $A_{n}$ for $n>1$, as well as all $D, E, F, G$-type diagrams, leaving only type $A_{1}$ among these.

Moreover, for types $B, C$, at most one simple root (the "last" one) is not killed by all $\lambda$ 's. If this root is long, then it is also killed by the key claim above (as a part of a $B_{2}$ ), and we are done.

2. First, $\lambda\left(s_{\alpha}^{2}\right)=\lambda\left(s_{\alpha}\right)^{2}=1$, whence $\lambda\left(s_{\alpha}\right)= \pm 1=1 \forall \alpha \in \Delta$, if $\operatorname{char}(R)=2$. This implies that $\lambda(w)=1=\varepsilon(w)$ for all $w \in W, \lambda \in \Gamma$. Next, Theorem 7.8 above tells us that if $\Pi$ has an element of order 4 , then $\Sigma_{\Pi}(\mathbf{h})=0$. Finally, if some $h_{i}$ has no " $\alpha_{s}$-contribution", then it is killed by all $\lambda$, by the previous part, so $\lambda(\mathbf{h})=0 \forall \lambda \in \Gamma$.

3. As we remarked after Theorem $7.10, \Pi=(\mathbb{Z} / 2 \mathbb{Z})^{k}$ in characteristic 2 , if $\Pi$ does not have an element of order 4. (Reason: $\Gamma=\{\varepsilon\} \times \mathfrak{h}^{W} \cong\left(\mathfrak{h}^{W},+\right)$ is a free $R$-module by the previous part, and $2 \Gamma=0$.)

We now perform the computation. For this, suppose that $h_{i}-c_{i} \alpha_{s}$ is in the $R$-span of $\{\alpha \in$ $\left.\Delta: \alpha \neq \alpha_{s}\right\}$ (note that in the case of $A_{1}$, the condition $h_{i} \in R \cdot \alpha_{s}$ is automatic). Then $\Sigma_{\Pi}(\mathbf{h})=\left(\prod_{i} c_{i}\right) \cdot \Sigma_{\Pi}\left(\alpha_{s}^{n}\right)$, so it suffices to compute $\Sigma_{\Pi}\left(\alpha_{s}^{n}\right)$.

If $\left\{\gamma_{i}\right\}$ is any set of generators (or $\mathbb{Z} / 2 \mathbb{Z}$-basis) for $\Pi$, then the desired equation actually holds if we sum over all nonnegative tuples $l_{i}$ that add up to $k$. Thus, the proof is similar to that of the key claim used to prove Theorem 7.10 above; simply note that if $I \subsetneq[k]$, then every $\prod_{j \in I} \gamma_{j}\left(\alpha_{s}^{\left|I_{j}\right|}\right)$ occurs with an even coefficient.

Finally, we prove the key claim.

Proof of the key claim. It helps to look at the pictures of these rank 2 root systems (drawn in [7, Chapter 3]). We use the $W$-invariance of $\left.\lambda\right|_{V} \forall \lambda \in \Gamma$.

Consider the system $A_{2}$, with simple roots $\alpha, \beta$. Given $\lambda \in \Gamma, \lambda(\alpha)=\lambda(\beta)=\lambda(\alpha+\beta)$, whence $\lambda(\alpha)=\lambda(\beta)=0$.

The root system $G_{2}$ has two subsystems of type $A_{2}$, whence each $\lambda$ must kill both subsystems.

Now consider $B_{2}$, with long root $\alpha$ and short root $\beta$. Clearly, $\beta+\alpha$ is another short root, whence $\lambda(\beta+\alpha)=\lambda(\beta)$, and we are done.

\subsection{The Reductive Case}

We conclude by mentioning what happens in the reductive case. This uses the results proved in the simple case above. Recall also that the notation for this situation was set when we defined degenerate affine Hecke algebras with trivial parameter earlier. This notation will be used freely here, without recalling it from above. 
Let $V^{\prime}$ be the direct sum of $V_{0}$ and the $R$-span of all the unique short simple roots $\alpha_{i \text {, short }}$ inside any of the simple components $V_{i}=\mathfrak{h}_{i}^{*}$ of the "correct" type (not $G_{2}$ ). Let the other simple roots in $\Delta$ span the $R$-submodule $V^{\prime \prime}$. Then $V=V^{\prime} \oplus V^{\prime \prime}$, and each $\lambda \in \Gamma$ kills $V^{\prime \prime}$. There now are two cases.

Case 1. $\operatorname{char}(R) \neq 2$. Then $\lambda$ in fact kills all $\alpha \in \Delta$, because $\lambda(\alpha)=\lambda\left(s_{\alpha}(\alpha)\right)=-\lambda(\alpha)$. This means that we are left with $V_{0}$, i.e., if for all $i, h_{i}-v_{0, i} \in \oplus_{j>0} V_{j}$ for some $v_{0, i} \in V_{0}$, then $\Sigma_{\Pi}(\mathbf{h})=\Sigma_{\Pi}\left(\prod_{i} v_{0, i}\right)$.

Next, recall that $\Sigma_{\Pi}=\Gamma_{W} \times\left(V^{*}\right)^{W}$, so we are reduced to the case of every $\lambda$ being represented (on $V_{0}$ ) by some element of $V_{0}^{*}=\left(V_{0}^{*}\right)^{W}$. We conclude this case by noting that some (partial) results on how to compute this were included in the previous section.

Case 2. $\operatorname{char}(R)=2$. Then $\lambda(w)=1$ for all $w, \lambda$, as seen above. Moreover, we are left only to consider the case of all $h_{i} \in V^{\prime}$. Now, $\Gamma=\varepsilon_{W} \times\left(V^{\prime}\right)^{*}$, whence any finite subgroup $\Pi=(\mathbb{Z} / 2 \mathbb{Z})^{k}$ for some $k$ (since it too is a $\mathbb{Z} / 2 \mathbb{Z}$-vector space). In this situation, Theorems 7.8 and 7.10 (and 9.3 as well) give us some information on how to compute $\Sigma_{\Pi}(\mathbf{h})$.

\section{An Example that Attains Any Value}

We conclude with examples where $\Sigma_{\Pi}(\mathbf{h})$ can take any value in $R$, if the $h_{i}$ 's are merely skew-primitive.

Example 12 (A skew-primitively generated algebra). By Proposition 5.5 above, if all $h_{i}$ 's are pseudo-primitive, then $\Sigma_{\Pi}(\mathbf{h})=0$ if $\operatorname{char}(R) \nmid|\Pi|$ —whereas if $\operatorname{char}(R)$ divides $|\Pi|$, then this case was analyzed in Section 7 above.

One can ask if such results hold in general, i.e., for products of skew-primitive elements. (Note by Theorem 5.6 that we need $\operatorname{char}(R) \nmid|\Pi|$.)

For the example that we now mention (for groups $\Pi$ of even order), one needs to assume the following:

1. $\operatorname{char}(R)>2^{n}$ and $\exp (\Pi)$, or $\operatorname{char}(R)=0$ and $R \supset \mathbb{Q}$; and

2. If $d=\exp (\Pi)$ is the exponent, then $d$ is even, and there exists a primitive $d$ th root of unity in $R$, say $z$.

Beyond this, given $n, \Pi$ (of even order), and $r \in R$, we will produce the desired Hopf algebra $\mathscr{H}$, a group of weights $\Pi \subset \Gamma \mathscr{H}$, and skew-primitive $h_{1}, \ldots, h_{n} \in H$, such that $\Sigma_{\Pi}(\mathbf{h})=r \in R$.

Given $\Pi$, use the Structure Theorem for finite Abelian groups to write $\Pi=\bigoplus_{i=1}^{k}\left(\mathbb{Z} / d_{i} \mathbb{Z}\right)$, with $d_{1}\left|d_{2}\right| \ldots \mid d_{k}=\exp (\Pi)$. Then $d_{k}$ is even, since $\Pi$ has even order. Now define $\mathscr{H}$ to be the commutative $R$-algebra freely generated as: $\mathscr{H}=R\left[R^{n}\right] \otimes R\left[\mathbb{Z}^{k}\right]$. In other words, $R$ is generated by $h_{1}, \ldots, h_{n}, g_{1}^{ \pm 1}, \ldots, g_{k}^{ \pm 1}$, with the relation that they all commute (and that the $g_{i}$ 's are invertible).

Now define the $g_{i}$ 's to be grouplike and $\Delta\left(h_{j}\right)=g_{k} \otimes h_{j}+h_{j} \otimes 1$. Also define (for all $i, j$ ):

$$
\varepsilon\left(g_{i}\right)=1, S\left(g_{i}\right)=g_{i}^{-1}, \varepsilon\left(h_{j}\right)=0, S\left(h_{j}\right)=-g_{k}^{-1} h_{j}
$$

Since $\mathscr{H}$ is freely generated, the set of weights of $\mathscr{H}$ is $R^{n} \times\left(R^{\times}\right)^{k}$. Since (it can be checked that) $\mathscr{H}$ is also a Hopf algebra, the group operation is:

$$
\begin{aligned}
& \left(a_{1}, \ldots, a_{n}, z_{1}, \ldots, z_{k}\right) *\left(a_{1}^{\prime}, \ldots, a_{n}^{\prime}, z_{1}^{\prime}, \ldots, z_{k}^{\prime}\right) \\
= & \left(a_{1}+z_{k} a_{1}^{\prime}, a_{2}+z_{k} a_{2}^{\prime}, \ldots, a_{n}+z_{k} a_{n}^{\prime}, z_{1} z_{1}^{\prime}, \ldots, z_{n}, z_{n}^{\prime}\right)
\end{aligned}
$$


We now produce the desired example. Define $\gamma_{j} \in \Gamma \mathscr{H}$ on generators by: $\gamma_{j}\left(g_{i}\right)=z^{\delta_{i j} d_{k} / d_{j}}$, and $\gamma_{j}\left(h_{i}\right)=0$ unless $j=k$. Moreover, $\gamma_{k}\left(h_{i}\right)=1$ for $i<k$, and $\gamma_{k}\left(h_{k}\right)=(1-z)^{n}|\Pi|^{-1} r^{\prime}$ for some $r^{\prime} \in R$ (which we define later, and which depends on $n$ ).

It is now easy to check that each $\gamma_{j}$ is of order $d_{j}$, and the $\gamma_{j}$ 's generate a subgroup of $\Gamma_{\mathscr{H}}$ isomorphic to $\Pi$. Moreover, $\gamma_{1}, \ldots, \gamma_{k-1}$ all kill $h_{1}, h_{2}, \ldots, h_{n}$. Adopting the notation of Proposition $4.2, \gamma_{j} \in \Gamma_{h_{i}}$ for $j<k$ and all $i$. Since $\Gamma_{h_{i}}$ is a subgroup of $\Gamma_{\mathscr{H}}$, hence $\Pi_{1}:=\left\langle\gamma_{1}, \ldots, \gamma_{k-1}\right\rangle \subset \Gamma_{h_{i}}$ for all $i$; by Proposition 4.2, $\Pi_{1} \subset \Gamma_{\mathbf{h}}$.

Now use Lemma 4.4 (noting that $\Pi_{1}$ is normal in the Abelian group $\Pi$ ); then $\Sigma_{\Pi}(\mathbf{h})=\left|\Pi / \Pi_{1}\right| \Sigma_{\Pi_{1}}(\mathbf{h})$. Use Theorem 6.3: $f_{i}=\frac{\gamma_{k}\left(h_{i}\right)}{\gamma_{k}\left(g_{k}\right)-1}$, so

$$
(-1)^{n} \prod_{i} f_{i}=(-1)^{n} \frac{(1-z)^{n}|\Pi|^{-1} r^{\prime}}{\prod_{i=1}^{n}(z-1)}=\frac{r^{\prime}}{|\Pi|}
$$

Moreover, $S=\left\{I \subset\{1,2, \ldots, n\}: g_{k}^{|I|}\right.$ is fixed by $\left.\Pi_{1}\right\}$, i.e., all subsets $I$ such that $d_{k}|| I \mid$. Since $d_{k}$ is even, this means that $(-1)^{|I|}=1 \forall I \in S$, whence

$$
\Sigma_{\Pi}(\mathbf{h})=\left|\Pi / \Pi_{1}\right| \Sigma_{\Pi_{1}}(\mathbf{h})=\frac{|\Pi|}{\left|\Pi_{1}\right|} \cdot\left|\Pi_{1}\right| \cdot \frac{r^{\prime}}{|\Pi|} \cdot \sum_{m \geq 0}\left(\begin{array}{c}
n \\
m d_{k}
\end{array}\right)=r^{\prime} \sum_{m \geq 0}\left(\begin{array}{c}
n \\
m d_{k}
\end{array}\right)
$$

By assumption (on $R$ ), the summation is a unit in $R$, so choosing $r^{\prime}$ suitably, one obtains any $r \in R$ as our answer.

Also note (e.g., by [17, Exercise 38, §1.2.6]), that the summation equals $\frac{1}{d_{k}} \sum_{l=0}^{d_{k}-1}\left(1+z^{l}\right)^{n}$.

\section{Acknowledgments}

I thank Susan Montgomery and Nicolás Andruskiewitsch for their comments and suggestions after reading a preliminary draft of this manuscript. I also thank the referees for their feedback, which helped improve the exposition of this paper.

\section{References}

1. Radford, D.E. The order of the antipode of a finite-dimensional Hopf algebra is finite. Am. J. Math. 1976, 98, 333-355.

2. Andruskiewitsch, N.; Schneider, H.-J. On the classification of finite-dimensional pointed Hopf algebras. Ann. Math. 2010, 171, 375-417.

3. Montgomery, S. Hopf Algebras and Their Actions on Rings; American Mathematical Society: Providence, RI, USA, 1993.

4. Abe, E. Hopf Algebras; Cambridge University Press: London, UK; New York, NY, USA, 1977.

5. Dascalescu, S.; Nastasescu, C.; Raianu, S. Hopf Algebras: An Introduction; Marcel-Dekker: New York, NY, USA, 2001.

6. Sweedler, M.E. Hopf Algebras; W.A. Benjamin: New York, NY, USA, 1969.

7. Humphreys, J.E. Introduction to Lie Algebras and Representation Theory; Springer-Verlag: New York, NY, USA, 1972. 
8. Jantzen, J.C. Lectures on Quantum Groups; American Mathematical Society: Providence, RI, USA, 1995.

9. Majid, S. A Quantum Groups Primer; Cambridge University Press: New York, NY, USA, 2002.

10. $\mathrm{Hu}, \mathrm{N}$. Quantum group structure associated to the quantum affine space. Algebra Colloq. 2004, 11, 483-492.

11. Hu, N. Quantum group structure of the $q$-deformed virasoro algebra. Lett. Math. Phys. 1998, 44, 99-103.

12. Hadfield, T.; Krähmer, U. On the Hochschild homology of quantum $S L(N)$. Comptes Rendus Math. 2006, 343, 9-13.

13. Khare, A. Functoriality of the BGG category $\mathcal{O}$. Commun. Algebra 2009, 37, 4431-4475.

14. Dascalescu, S.; Iovanov, M.C.; Nastasescu, C. Quiver algebras, path coalgebras, and co-reflexivity. Pacific J. Math. arXiv:1208.4410.

15. Alperin, J.L.; Bell, R.B. Groups and Representations; Springer-Verlag: New York, NY, USA, 1995.

16. Cherednik, I. Integration of quantum many-body problems by affine knizhnik-zamolodchikov equations. Adv. Math. 1994, 106, 65-95.

17. Knuth, D.E. The Art of Computer Programming, Volume 1: Fundamental Algorithms; Addison-Wesley: Boston, MA, USA, 1997.

(c) 2012 by the author; licensee MDPI, Basel, Switzerland. This article is an open access article distributed under the terms and conditions of the Creative Commons Attribution license (http://creativecommons.org/licenses/by/3.0/). 